Publ. RIMS, Kyoto Univ.

15 (1979), 519-550

\title{
Cauchy Problem for Non-Strictly Hyperbolic Systems
}

By

\author{
Kunihiko KaJITANI*
}

\section{Introduction}

We consider the Cauchy problem for non-strictly hyperbolic systems with characteristic roots of constant multiplicity.

We shall indicate some sufficient condition in order that the Cauchy problem is well posed in $C^{\infty}$ class. In particular we shall give a necessary and sufficient condition for first order hyperbolic systems.

We introduce the following notation,

$$
\begin{aligned}
& x=\left(x_{0}, x^{\prime}\right)=\left(x_{0}, x_{1}, \cdots, x_{n}\right) \in \mathbb{R}^{n+1}, \\
& \xi=\left(\xi_{0}, \xi^{\prime}\right)=\left(\xi_{0}, \xi_{1}, \cdots, \xi_{n}\right) \in \mathbb{R}^{n+1}, \\
& D=\left(D_{0}, D^{\prime}\right)=\left(D_{0}, D_{1}, \cdots, D_{n}\right), \\
& D_{j}=-i \frac{\partial}{\partial x_{j}} .
\end{aligned}
$$

Let $G$ be an open domain in $\boldsymbol{R}^{n+1}$. Let

$$
P(x, D) u=\sum_{|\alpha| \leq m} A_{\alpha}(x) D^{\alpha} u,
$$

where $u={ }^{t}\left(u_{1}, \cdots, u_{N}\right), \quad A_{\alpha}(x)$ is a $N \times N$-matrix of elements in $C^{\infty}(G)$,

$$
\begin{aligned}
& P(x, \xi)=\sum_{|\alpha| \leq m} A_{\alpha}(x) \xi^{\alpha}, \quad P_{s}(x, \xi)=\sum_{|\alpha|=s} A_{\alpha}(x) \xi^{\alpha}, \\
& \alpha=\left(\alpha_{0}, \alpha_{1}, \cdots, \alpha_{n}\right), \quad|\alpha|=\alpha_{0}+\cdots+\alpha_{n}, \\
& D^{\alpha}=D_{0}^{\alpha_{0}} \cdots D_{n}^{\alpha_{n}}, \quad \xi^{\alpha}=\xi_{0}^{\alpha_{0}} \ldots \xi_{n}^{\alpha_{n}} .
\end{aligned}
$$

Let $\widehat{x}$ be in $G$ and $G(\widehat{x})$ a neighborhood of $\widehat{x}$.

Communicated by S. Matsuura, April 16, 1977.

* Department of Mathematics, University of Tsukuba. 
Consider the Cauchy problem

$$
\left\{\begin{array}{l}
P u=f \text { in } G(\widehat{x}) \cap\left\{x_{0}>\widehat{x}_{0}\right\}, \\
D_{0}^{j} u=g_{j} \text { on } G(\widehat{x}) \cap\left\{x_{0}=\widehat{x}_{0}\right\}, j=0, \cdots, m-1,
\end{array}\right.
$$

where $f$ and $g_{j}$ are vector valued functions.

Definition 1. The Cauchy problem (1) for $P$ is said to be well posed at $\hat{x} \in G$, if the following conditions hold:

(E) There exists $G(\hat{x}) \subset G$, a neighborhood of $\hat{x}$, such that for any $f(x) \in C^{\infty}(G(\hat{x}))$ and $g_{j} \in C^{\infty}\left(G(\hat{x}) \cap\left\{x_{0}=\widehat{x}_{0}\right\}\right)$, there is a function $u(x) \in C^{\infty}(G(\widehat{x}))$ satisfying $(1)$.

(U) If for any $G(\hat{x}) \subset G$, a neighborhood of $\hat{x}$, there exists $\widetilde{G}(\widehat{x}) \subset G(\widehat{x})$, a neighborhood of $\widehat{x}$ such that if $u \in C^{\infty}(G(\widehat{x}))$ satisfies (1) and $P u=0$ in $\widetilde{G}(\widehat{x}) \cap\left\{x_{0}>\widehat{x}_{0}\right\}$ and supp $u \subset\left\{x_{0}>\widehat{x}_{0}\right\}$, then $u=0$ in $\widetilde{G}(\widehat{x}) \cap\left\{x_{0}>\widehat{x}_{0}\right\}$.

If for any $\widehat{x} \in G$, the Cauchy problem (1) for $P$ is well posed at $\widehat{x}$, then $P$ is said to be well posed in $G$.

We say that $P$ is a hyperbolic system of constant multiplicity, if it holds

$$
\operatorname{det} P_{m}(x, \xi)=\prod_{l=1}^{r}\left(\xi_{0}+\lambda^{(l)}\left(x, \xi^{\prime}\right)\right)^{\nu_{l}}
$$

here $\nu_{l}$ is an integer, and $\lambda^{(l)}$ are real valued functions in $C^{\infty}(G \times$ $\left.\left\{\boldsymbol{R}^{n}-0\right\}\right), \sum_{l=1}^{r} \nu_{l}=m N$ and $\lambda^{(l)} \neq \lambda^{(j)}$ for $l \neq j$.

We start with the Cauchy problem for the hyperbolic systems with a diagonal principal part. We say that $P$ is a hyperbolic system with diagonal principal part of constant multiplicity, if the principal part of $P$ has the form,

$$
P_{m}(x, \xi)=\prod_{l=1}^{r}\left(\xi_{0}+\lambda^{(l)}\left(x, \xi^{\prime}\right)\right)^{\nu_{l}} I,
$$

where $I$ is the identity matrix and $\lambda^{(l)}\left(x, \xi^{\prime}\right)$ are real valued functions in $C^{\infty}\left(G \times\left\{\boldsymbol{R}^{n}-0\right\}\right), \sum_{l=1}^{r} \nu_{l}=m N$.

Denote the phase function of $P$ by $\varphi^{(l)}(x)$, that is

$$
\varphi_{x_{0}}^{(l)}+\lambda^{(l)}\left(x, \varphi_{x^{\prime}}^{(l)}\right)=0, \quad \varphi_{x^{\prime}}^{(l)} \neq 0 .
$$


Definition 2. Let $P$ be a hyperbolic system with diagonal principal part of constant multiplicity. Let $P_{t}^{s}$ be the $(s, t)$-element of $P$. It is said that $P$ satisfies the Levi's condition in $G$ if there exist integers $n_{s}^{(l)}, l=1, \cdots, r, s=1, \cdots, N$ such that for any phase function $\varphi^{(l)}(x)$ and for any scalar function $w \in C_{0}^{\infty}(G)$

$$
e^{-i \rho \varphi(l)} P_{t}^{s}\left(e^{i \rho \varphi(l)} w\right)=0\left(\rho^{m-\nu+n_{t}(l)-n_{s}(l)}\right), \quad(\rho \rightarrow \infty),
$$

for $s, t=1, \cdots, N, l=1, \cdots, r$.

Remark. This condition was suggested by Leray-Ohya [5]. When $N=1$, this is the usual Levi's condition (c.f. [2], [6]). If we can choose $n_{t}^{(l)}=n_{s}^{(l)}$, our condition is same one as Gourdin [3] and VaillantBersin [9].

Theorem 1. Let $P$ be a hyperbolic system with diagonal principal part of constant multiplicity. If $P$ satisfies the Levi's condition $(L)$ in $G$ then $P$ is well posed in $G$.

Remark. The condition (L) is not a necessary condition in order that $P$ is well posed in $G$. For example

$$
P=\left(\begin{array}{cc}
D_{0}^{2} & 0 \\
0 & D_{0}^{2}
\end{array}\right)+\left(\begin{array}{cc}
D_{1} & D_{1} \\
-D_{1} & -D_{1}
\end{array}\right)
$$

is well posed in $\mathbb{R}^{2}$. But we can never choose integers satisfying (L). We shall investigate the necessary condition in the section 3 (Theorem 3. 2).

We next consider a hyperbolic system of constant multiplicity. We say that $Q$ is a cofactor system of $P$, if the principal symbol of $Q$ is the cofactor matrix of $P_{m}(x, \xi)$. Then if $P$ is a hyperbolic system of constant multiplicity, $P Q$ and $Q P$ are both hyperbolic systems with diagonal principal part of constant multiplicity.

We obtain the following theorem as a corollary of Theorem 1.

Theorem 2. Let $P$ be a hyperbolic system of constant multiplicity. If there exist two cofactor systems $Q, R$ of $P$ such that $P Q$ 
and RP satisfy the Levi's condition in $G$, then $P$ is rell posed in G.

Example (Petkov). Let

$$
P=I D_{0}+\left(\begin{array}{ccc}
0 & 1 & 0 \\
& 0 & 0 \\
& & 0
\end{array}\right) D_{1}+\left(\begin{array}{lll}
b_{11} & b_{12} & b_{13} \\
b_{21} & b_{22} & b_{23} \\
b_{31} & b_{32} & b_{33}
\end{array}\right)
$$

If $b_{21}(x)=b_{31}(x)=0$ or $b_{21}(x)=b_{23}(x)=0$ is valid, then $P$ is well posed. Set

$$
Q=I D_{0}^{2}-\left(\begin{array}{ccc}
0 & 1 & 0 \\
& 0 & 0 \\
0 & & 0
\end{array}\right) D_{0} D_{1}
$$

Then $P Q$ satisfies the Levi's condition (L), when we choose

$$
\begin{aligned}
\left(n_{1}, n_{2}, n_{3}\right) & =(0,1,1), \quad \text { if } \quad b_{21}=b_{31}=0 \\
& =(0,1,0), \quad \text { if } \quad b_{21}=b_{23}=0 .
\end{aligned}
$$

We shall examine more precisely a first order hyperbolic system of constant multiplicity, that is, $m=1$,

$$
P(x, D)=I D_{0}+\sum_{j=1}^{n} A_{j}(x) D_{j}+B(x),
$$

where $A_{j}(x)$ and $B(x)$ are $N \times N$ matrices of elements in $C^{\infty}(G)$. Moreover we assume

$$
\operatorname{rank}\left(\lambda^{(l)}\left(x, \xi^{\prime}\right) I-\sum_{j=1}^{n} A_{j}(x) \xi_{j}\right)=N-1, l=1, \cdots, r .
$$

Then we can construct a pseudo-differential operator $N\left(x, D^{\prime}\right)$ of order zero with respect to $D^{\prime}$, which transforms microlocally $P$ into $\widetilde{P}$ such that

$$
P(x, D) N\left(x, D^{\prime}\right)=N\left(x, D^{\prime}\right) \widetilde{P}(x, D),
$$

where

$$
\widetilde{P}(x, D)=\left(\begin{array}{ccc}
\widetilde{P}^{(1)}(x, D) & 0 \\
& \ddots & \widetilde{P}^{(r)}(x, D)
\end{array}\right),
$$




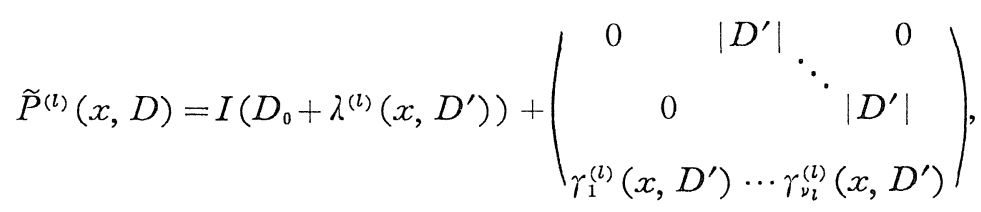

$$
\begin{aligned}
& l=1, \cdots, r \text {, }
\end{aligned}
$$

$\gamma_{k}^{(l)}\left(x, D^{\prime}\right)$ is a pseudo-differential operator of order zero with respect to $D^{\prime}$ (c.f. Proposition 2.2).

Then we obtain,

Theorem 3. Let $P$ be a first order hyperbolic system of constant multiplicity. Assume that the condition $(R)$ is valid. Then $P$ is reell posed in $G$, if and only' if

$$
\operatorname{order} \gamma_{k}^{(l)}\left(x, D^{\prime}\right) \leq k-\nu_{l},
$$

for $k=1, \cdots, \nu_{l}-1, l=1, \cdots, r$.

Remark. We note that our theorem holds, if we assume instead of $(\mathrm{R})$,

$$
\operatorname{rank}\left(\lambda^{(l)}\left(x, \xi^{\prime}\right) I-\sum A_{j}(x) \xi_{j}\right)=\left\{\begin{array}{l}
N-1 \\
\text { or } \\
N-\nu_{l}
\end{array}\right.
$$

In [7] Petkov has given the Levi's condition as follows; $P$ satisfies the condition $\left(\mathrm{L}_{2}\right)$, if for any $w(x) \in C_{0}^{\infty}(G)$ and for any phase function $\varphi^{(l)}(x)$, there exist vector valued functions $V_{k}^{(l)}\left(x, \varphi^{(l)}, w\right), \quad(k=1, \cdots$, $\left.\nu_{l}-1\right)$, such that

$$
\begin{gathered}
e^{-i \rho \varphi(l)} P\left[e^{i \rho \varphi(l)}\left\{w(x) N_{1}^{(l)}\left(x, \varphi_{x^{\prime}}^{(l)}\right)+\sum_{k=1}^{\nu_{l}-1} \rho^{-k} V_{k}\left(x, \varphi^{(l)}, w\right)\right\}\right] \\
=0\left(\rho^{1-\nu}\right),(\rho \rightarrow \infty),
\end{gathered}
$$

where $N_{1}{ }^{(l)}\left(x, \xi^{\prime}\right)$ is a right null vector of the matrix $\left(\lambda^{(l)}\left(x, \xi^{\prime}\right) I\right.$ $\left.-\sum A_{j}(x) \xi_{j}\right)$.

Theorem 4. Let $P$ be a first order hyperbolic system of constant multiplicity. Assume that $(R)$ is valid. Then our condition $\left(L_{1}\right)$ is equivalent to $\left(L_{2}\right)$ given by Petkov. 
In [8] Petkov constructs the parametrix of $P$ under the condition $\left(\mathrm{L}_{2}\right)$. When $\nu_{l}=2$, Yamahara has derived the condition $\left(\mathrm{L}_{1}\right)$ in $[10]$.

\section{§ 1. Systems with Dagonal Principal Part}

We shall construct a parametrix of the Cauchy problem for $P$, a hyperbolic system with diagonal principal part of constant multiplicity satisfying the condition (L). It follows from the existence of the parametrix that the Cauchy problem for $P$ is well posed. For, if $P$ satisfies (L), then $P^{(*)}$ the adjoint operator of $P$ does so and since the condition $(\mathrm{L})$ is invariant under a transform of coordinate variables, a solution of the Cauchy problem for $P$ has a finite propagation speed and therefore the Cauchy problem for $P$ has the local uniqueness (c.f. [1]).

Denote by $L^{m}(G)$ the class of pseudo-differential operators of which symbol is developed asymptotically,

$$
a(x, \xi)=\sum_{j=0}^{\infty} a_{j}(x, \xi),
$$

where $a_{j}(x, \hat{\xi})$ are homogeneous degree $m-j$ in $\hat{\xi}$ and polynomials with respect to $\xi_{0}$.

Let $a(x, D)$ be in $L^{m}(G)$ and $w$ in $C^{\infty}(G)$. Then we can develop asymptotically

$$
e^{-i \rho \varphi} a(x, D)\left(e^{i \rho \varphi} w\right)=\sum_{j=0}^{\infty} \rho^{m-j} \sigma_{j}(\varphi, a)
$$

where $\sigma_{j}(\varphi, a)$ are the differential operators of which principal part is given by

$$
\sum_{|a|=j}\left(\left(\frac{\partial}{\partial \xi}\right)^{\alpha} a_{0}\right)\left(x, \varphi_{x}\right) D^{\alpha} / \alpha !
$$

in particular, we have

$$
\begin{aligned}
\sigma_{0}(\varphi, a)= & a_{0}\left(x, \varphi_{x}\right) \\
\sigma_{1}(\varphi, a)= & \sum_{j=0}^{n}\left(\frac{\partial}{\partial \xi_{j}} a_{0}\right)\left(x, \varphi_{x}\right) D_{j}+a_{1}\left(x, \varphi_{x}\right) \\
& +i \sum_{|\alpha|=2}\left(\left(\frac{\partial}{\partial \xi}\right)^{\alpha} a_{0}\right)\left(x, \varphi_{x}\right) D^{\alpha} / \alpha ! .
\end{aligned}
$$


Let us consider $P$, a hyperbolic system with diagonal principal part,

$$
a(x, D)=\prod_{l=1}^{r} q^{(l)}(x, D)^{\nu_{l}},
$$

where $q^{(l)}(x, \xi)=\xi_{0}+\lambda^{(l)}\left(x, \xi^{\prime}\right), \sum_{l=1}^{r} \nu_{l}=m$. We note that $a(x, D)$ satisfies,

$$
e^{-i \rho \varphi(l)} a(x, D)\left(e^{i \rho \varphi(l)} w\right)=0\left(\rho^{m-\nu l}\right)
$$

for $l=1, \cdots, r$, where $\varphi^{(l)}(x)$ is a phase function corresponding to $q^{(l)}$. Let $b_{t}^{s}(x, D)$ be in $L^{m-1}(G), s, t=1, \cdots, N$.

Consider the following Cauchy problem;

$$
\begin{aligned}
& a(x, D) u^{s}(x)+\sum_{t=1}^{N} b_{t}^{s}(x, D) u^{t}(x)=0, s=1, \cdots, N, \\
& \left.D_{0}^{j} u^{s}\right|_{x_{0}=0}=e^{i\left\langle x^{\prime}, \xi^{\prime}\right\rangle} g_{j}^{s}\left(x, \xi^{\prime}\right), \quad j=0,1, \cdots, m-1,
\end{aligned}
$$

where

$$
g_{j}^{s}\left(x, \xi^{\prime}\right)=\sum_{p=0}^{\infty} g_{j p}^{s}\left(x, \xi^{\prime}\right)\left|\xi^{\prime}\right|^{r_{0}-p}
$$

$g_{j p}^{s}\left(x, \xi^{\prime}\right)$ are homogeneous degree zero in $\xi^{\prime}$. Let us choose a phase function $\varphi^{(l)}\left(x, \xi^{\prime}\right)$ such that

$$
\begin{aligned}
& q^{(l)}\left(x, \varphi_{x^{\prime}}^{(l)}\right)=0 \\
& \left.\varphi^{(l)}\right|_{x_{0}=0}=\left\langle x^{\prime}, \xi^{\prime}\right\rangle /\left|\xi^{\prime}\right| .
\end{aligned}
$$

Then we have

Theorem 1. 1. Let $b_{s}^{t}(x, D)$ be in $L^{m-1}(G)$. Assume that $b_{s}^{t}(x, D)$ satisfies the condition $(L)$, that is, for any $w \in C^{\infty}(G)$,

$$
e^{-i \rho \varphi(l)} b_{t}^{s}(x, D)\left(e^{i \rho \varphi(l)} w\right)=0\left(\rho^{m-\nu_{l}+n_{t}(l)-n_{s}(l)}\right),
$$

then the Cauchy problem (1.2) has an asymptotic solution $\left(u^{s}\right)$, $s=1, \cdots, N$ such that,

$$
u^{s}\left(x, \xi^{\prime}\right)=\sum_{l=1}^{r} \sum_{j=0}^{\infty} e^{i \rho \varphi(l)}\left(x, \xi^{\prime}\right) \rho^{m_{0}-\nu_{l}-n_{s}(l)-j} u_{l j}^{s}\left(x, \xi^{\prime}\right),
$$

where $\rho=\left|\xi^{\prime}\right|, m_{0}=\gamma_{0}+\max _{l, t} n_{t}^{(l)}$, and $u_{l j}^{s}\left(x, \xi^{\prime}\right)$ are homogeneous degree zero in $\xi^{\prime}$. 
Proof. We can write by virtue of (1.1) and (1.4),

$$
\begin{aligned}
e^{-i \rho \varphi(l)} & a(x, D)\left(e^{i \rho \varphi(l)} w\right) \\
& =\sum_{p=0}^{\infty} \sigma_{p}\left(\varphi^{(l)}, a\right) \rho^{m-p} w \\
& =\sum_{p=0}^{\infty} \sigma_{p+\nu_{l}}\left(\varphi^{(l)}, a\right) \rho^{m-\nu_{l}-p} w,
\end{aligned}
$$

and

$$
\begin{aligned}
e^{-i \rho \varphi(l)} & b_{t}^{s}(x, D)\left(e^{i \rho \varphi(l)} w\right) \\
& =\sum_{p=0}^{\infty} \sigma_{p}\left(\varphi^{(l)}, b_{t}^{s}\right) \rho^{m-1-p} w \\
& =\sum_{p=0}^{\infty} \sigma_{p+\nu_{l}-1+n_{s}(l)-n_{t}(l)}\left(\varphi^{(l)}, b_{t}^{s}\right) \rho^{m-\nu_{l}+n_{t}(l)-n_{s}(l)-p} w .
\end{aligned}
$$

Inserting $u^{s}\left(t, \xi^{\prime}\right)$ of (1.5) into (1.2), we obtain

$$
\begin{gathered}
\sum_{l=1}^{r} \sum_{j, p=0}^{\infty} e^{i \rho \varphi(l)} \rho^{m+m_{0}-n_{s}(l)-p-j}\left\{\sigma_{p+\nu_{l}}\left(\varphi^{(l)}, a\right) u_{l_{j}}^{s}\right. \\
\left.+\sum_{t=1}^{N} \sigma_{p+\nu_{l}-1+n_{s}(l)-n_{t}(l)}\left(\varphi^{(l)}, b_{t}^{s}\right) u_{l j}^{t}\right\}=0
\end{gathered}
$$

for $s=1, \cdots, N$. Hence we have

$$
\sum_{j+p=k}\left\{\sigma_{p+\nu_{l}}\left(\varphi^{(l)}, a\right) u_{l_{j}}^{s}+\sum_{t=1}^{N} \sigma_{p+\nu_{l}-1+n_{g}(l)-n_{t}(l)}\left(\varphi^{(l)}, b_{t}^{s}\right) u_{l j}^{t}\right\}=0
$$

for $l=1, \cdots, r, s=1, \cdots, N, k=0,1, \cdots$, that is,

$$
\sigma_{\nu_{l}}\left(\varphi^{(l)}, a\right) u_{l k}^{s}+\sum_{t=1}^{N} \sigma_{\nu_{l}-1+n_{s}(l)-n_{t}(l)}\left(\varphi^{(l)}, b_{t}^{s}\right) u_{l k}^{t}=f_{l k}^{s}
$$

where

$$
f_{l k}^{s}=-\sum_{\substack{j+p=k \\ j<k}} \sigma_{p+\nu_{l}}\left(\varphi^{(l)}, a\right) u_{l j}^{s}+\sum_{i=1}^{N} \sigma_{p+\nu_{l}-1+n_{g}(l)-n_{t}(l)}\left(\varphi^{(l)}, b_{t}^{s}\right) u_{l j}^{t} .
$$

From the initial condition of (1.2) it follows that

$$
\begin{aligned}
\left.D_{0}^{q} u^{s}\right|_{x_{0}=0} & =\left.\sum_{i=1}^{r} e^{i\left\langle x^{\prime}, \xi^{\prime}\right\rangle} \sum_{j, p=0}^{\infty} \sigma_{p}\left(\varphi^{(l)}, D_{0}^{q}\right) \rho^{q+m_{0}-n_{s}(l)-p-j} u_{i j}^{s}\right|_{x_{0}=0} \\
& =e^{i\left\langle x^{\prime}, \xi^{\prime}\right\rangle} \sum_{k=0}^{\infty} g_{q k}^{s}\left(x, \xi^{\prime}\right) \rho^{\gamma_{0}-k}
\end{aligned}
$$

Put $m_{s}^{(l)}=m_{0}-\gamma_{0}-n_{s}^{(l)}$. Then we have 


$$
\left.\sum_{l=1}^{r} \sum_{p+j=k+m_{s}(l)+q} \sigma_{p}\left(\varphi^{(l)}, D_{0}^{q}\right) u_{l j}^{s}\right|_{x_{0}=0}=g_{q k}^{s} .
$$

Noting that the principal part of $\sigma_{q}\left(\varphi^{(l)}, D_{0}^{q}\right)$ is $\left(\begin{array}{c}q \\ p\end{array}\right)\left(\varphi_{x_{0}}\right)^{q-p} D_{0}^{p}$, we have

$$
\begin{gathered}
\sum_{l=1}^{r} \sum_{p=0}^{q}\left\{\left(\begin{array}{l}
q \\
p
\end{array}\right)\left(\varphi_{x_{0}}^{(l)}\right)^{q-p} D_{0}^{p} u_{l k+m_{s}(l)-p}^{s}+B_{q p}^{(l)}\left(x, D_{0}\right) u_{l k+m_{s}(l)-p}^{s}\right\}_{x_{0}=0} \\
=g_{q k-q}^{s},
\end{gathered}
$$

here $B_{q p}^{(l)}\left(x, D_{0}\right)$ are of order $p-1$. Since $\left.\varphi_{x_{0}}^{(l)}\right|_{x_{0}=0}=-\lambda^{(l)}\left(0, x, \xi^{\prime} /\left|\xi^{\prime}\right|\right)$, $l=1, \cdots, r$, are distinct, the determinant of Van der Monde $\left\{\left(\begin{array}{l}q \\ p\end{array}\right)\left(\varphi_{x_{0}}^{(l)}\right)^{q-p}\right.$, $\left.q=0,1, \cdots, m-1, p=0,1, \cdots, \nu_{l}-1, l=1, \cdots, r,\right\}$ is not zero. Hence we can solve (1.7) with respect to $\left\{D_{0}^{p} u_{l k+m_{s}(l)-p}^{s}\right\}, p=0,1, \cdots, \nu_{l}-1, l=1$, $\cdots, r$, for any $k$, where $u_{l k}^{s}=0$ if $k<0$. Therefore we can solve (1.6) and (1.7) successively by use of the following lemma. For, we have

$$
\sigma_{\nu_{l}}\left(\varphi^{(l)}, a\right)=\sum_{k=0}^{\nu_{l}} H_{l}(x, D)^{\nu_{l}-k} a_{k}^{(l)}(x), \quad a_{0}^{(l)}(x) \neq 0,
$$

and

$$
\sigma_{\nu_{l}-1+n_{s}(l)-n_{t}(l)}\left(\varphi^{(l)}, b_{t}^{s}\right)=\sum_{k=0}^{\nu_{l}-1+n_{g}(l)-n_{t}(l)} B_{t k}^{s}(x) H_{l}(x, D)^{k},
$$

where $H_{l}(x, D)=D_{0}+\sum_{j=1}^{n} \lambda_{\xi_{j}}^{(l)}\left(x, \varphi_{x^{\prime}}^{(l)}\right) D_{j}$.

Lemma 1. 2. (c.f. [1]). Let $b(x, D)$ be in $L^{m-1}(G)$ and $\varphi^{(l)}$ be a phase function satisfying (1.3). Assume that for any phase function $\varphi^{(l)}$ and for any $w \in C_{0}(G), \rho=\left|\xi^{\prime}\right|$,

$$
e^{-i \rho \varphi(t)} b(x, D)\left(e^{i \rho \varphi(l)} w\right)=0\left(\rho^{m-\nu}\right),
$$

then we obtain

$$
\sigma_{\nu-1}\left(\varphi^{(l)}, b\right)=\sum_{k=0}^{\nu-1} b_{\nu k}^{(l)}(x) H_{\imath}(x, D)^{k},
$$

where

$$
H_{l}(x, D)=D_{0}+\sum_{j=1}^{n} \lambda_{\xi_{j}}^{(l)}\left(x, \varphi_{x^{\prime}}^{(l)}\right) D_{j}
$$

Proof. We transform coodinate variables $x^{\prime}=x^{\prime}(t, z), x_{0}=t$, such that 


$$
\varphi^{(l)}\left(t, x^{\prime}\left(t, z^{\prime}\right), \xi^{\prime}\right)=\left\langle z^{\prime}, \xi^{\prime}\right\rangle /\left|\xi^{\prime}\right|
$$

that is, $x^{\prime}\left(t, z^{\prime}\right)$ is a solution;

$$
\begin{aligned}
& \frac{d}{d t} x^{\prime}\left(t, z^{\prime}\right)=\lambda_{\xi^{\prime}}^{(l)}\left(t, x^{\prime}\left(t, z^{\prime}\right), \varphi_{x^{\prime}}^{(l)}\right) \\
& x^{\prime}\left(0, z^{\prime}\right)=z^{\prime} .
\end{aligned}
$$

Then

(1. 9) $\quad D_{t}\left(w\left(t, x^{\prime}\left(t, z^{\prime}\right)\right)=\left.\left(D_{t}+\sum \lambda_{\xi_{j}}^{(l)} D_{j}\right) w\right|_{x=\left(t x^{\prime}\left(t, z^{\prime}\right)\right)}\right.$

and

$$
\begin{aligned}
& \left.e^{-i \rho \varphi(l)} b(x, D)\left(e^{i \rho \varphi(l)} w\right)\right|_{x=\left(t, x^{\prime}\left(t, z^{\prime}\right)\right)} \\
& \quad=e^{-i\left\langle z^{\prime}, \xi^{\prime}\right\rangle} \tilde{b}\left(t, z, D_{t}, D_{z}\right)\left(e^{i\left\langle z, \xi^{\prime}\right\rangle} w\left(t, x^{\prime}\left(t, z^{\prime}\right)\right)=0\left(\rho^{m-\nu}\right), \quad \rho=\left|\xi^{\prime}\right| .\right.
\end{aligned}
$$

Hence we have

$$
\begin{aligned}
\left.\sigma_{\nu-1}\left(\varphi^{(l)}, b\right)\right|_{x=\left(t, x^{\prime}\left(t, z^{\prime}\right)\right)} & =\sigma_{\nu-1}\left(\left\langle z^{\prime}, \xi^{\prime} /\left|\xi^{\prime}\right|\right\rangle, \tilde{b}\right) \\
& =\sum_{k=0}^{\nu-1} \tilde{b}_{\nu k}^{(l)}\left(t, z^{\prime}\right) D_{t}^{k},
\end{aligned}
$$

which implies (1.8) with (1.9).

Thus we have proved Theorem 1.1 which implies Theorem 1 and Theorem 2 in the introduction.

\section{§ 2. First Order Systems}

Consider the first order system,

$$
P=I D_{0}+\sum_{j=1}^{n} A_{j}(x) D_{j}+B(x),
$$

here $A_{j}(x)$ and $B(x)$ are $N \times N$ matrices of $C^{\infty}(G)$-elements. Set

$$
A\left(x, \xi^{\prime}\right)=\sum_{j=1}^{n} A_{j}(x) \xi_{j} .
$$

and

$$
M^{(l)}\left(x, \xi^{\prime}\right)=A\left(x, \xi^{\prime}\right)-\lambda^{(l)}\left(x, \xi^{\prime}\right) I .
$$

We suppose that 


$$
\left\{\begin{array}{l}
\operatorname{det}\left(\xi_{0}+A\left(x, \xi^{\prime}\right)\right)=\prod_{l=1}^{r}\left(\xi_{0}+\lambda^{(l)}\left(x, \xi^{\prime}\right)\right)^{\nu_{l}}, \\
\quad\left(\nu_{l} ; \text { positive integers }\right), \\
\operatorname{rank} M^{(l)}\left(x, \xi^{\prime}\right)=N-1,(l=1, \cdots, N) .
\end{array}\right.
$$

Lemma 2. 1 ([4]). Under the assumption (2.1), for any $\left(\hat{x}, \hat{\xi}^{\prime}\right)$ $\in G \times\left\{\boldsymbol{R}^{n}-0\right\}$, there exists a conic neighborhood $V\left(\hat{x}, \xi^{\prime}\right)$ and a matrix $N_{0}\left(x, \xi^{\prime}\right) \in C^{\infty}\left(V\left(\widehat{x}, \xi^{\prime}\right)\right)$ such that

$$
\begin{aligned}
& A\left(x, \xi^{\prime}\right) N_{0}\left(x, \xi^{\prime}\right)=N_{0}\left(x, \xi^{\prime}\right) \Lambda_{0}\left(x, \xi^{\prime}\right), \\
& \Lambda_{0}\left(x, \xi^{\prime}\right)=\left(\begin{array}{cc}
\lambda^{(l)}\left(x, \xi^{\prime}\right) I+C^{(l)} \mid \xi^{\prime} ! & 0 \\
0 & \dot{\lambda}^{(l)}\left(x, \xi^{\prime}\right) I+C^{(l)}\left|\xi^{\prime}\right|
\end{array}\right) \text {, }
\end{aligned}
$$

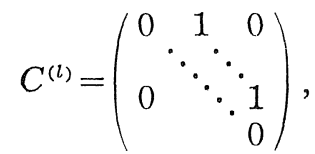

and $N_{0}\left(x, \xi^{\prime}\right)$ is homogeneous of degree zero and its determinant does not vanish for $\left(x, \xi^{\prime}\right) \in V\left(\widehat{x}, \hat{\xi}^{\prime}\right)$.

Proposition 2.2. Under the assumption (2.1), for any ( $\left.\widehat{x}, \hat{\xi}^{\prime}\right)$ $\in G \times\left\{\boldsymbol{R}^{n+1} \backslash 0\right\}$, there exists a pseudo-differential operator $N\left(x, D^{\prime}\right)$ of order zero such that

$$
P(x, D) N\left(x, D^{\prime}\right)=N\left(x, D^{\prime}\right) \widetilde{P}(x, D),\left(\bmod L^{-\infty}(G)\right),
$$

$$
\widetilde{P}(x, D)=I D_{0}+\left(\begin{array}{cccc}
\Lambda^{(1)}\left(x, D^{\prime}\right) & & \\
& 0 & \ddots & \\
& & \ddots & 0 \\
& & & \Lambda^{(r)}\left(x, D^{\prime}\right)
\end{array}\right),
$$

symbol of $\Lambda^{(l)}=\lambda^{(l)}\left(x, \xi^{\prime}\right) I+C^{(l)}\left|\xi^{\prime}\right|$

$$
+\left(\begin{array}{c}
0 \\
\gamma_{1}^{(l)}\left(x, \xi^{\prime}\right) \cdots \gamma_{\nu_{l}}^{(l)}\left(x, \xi^{\prime}\right)
\end{array}\right)
$$

for $\left(x, \xi^{\prime}\right) \in V\left(\hat{x}, \hat{\xi}^{\prime}\right)$, a conic neighborhood of $\left(\widehat{x}, \hat{\xi}^{\prime}\right)$, here $\gamma_{k}^{(l)}\left(x, \xi^{\prime}\right)$ $\in C^{\infty}\left(V\left(\widehat{x}, \hat{\xi}^{\prime}\right)\right)$ and $C^{(l)}$ is $\nu_{l} \times \nu_{l}$-Jordan's matrix of rank $\nu_{l}-1$.

Proof. We shall seek $N\left(x, D^{\prime}\right)$ such that, 


$$
N\left(x, D^{\prime}\right)=\sum_{j=0}^{\infty} N_{j}\left(x, D^{\prime}\right)
$$

here $N_{j}\left(x, \xi^{\prime}\right)$, the symbol of $N_{j}\left(x, D^{\prime}\right)$ is homogeneous degree $-j$ in $\xi^{\prime}$. Then we can write the symbol of $P N$ and $N P$,

$$
\begin{aligned}
(P N)\left(x, \xi^{\prime}\right)= & \sum_{j=0}^{\infty}\left(\xi_{0}+A\left(x, \xi^{\prime}\right)\right) N_{j}\left(x, \xi^{\prime}\right) \\
& +P(x, D) N_{j-1}\left(x, \xi^{\prime}\right)
\end{aligned}
$$

and

$$
\begin{aligned}
(N P)(x, \xi) & =\sum_{\alpha}\left(\frac{\partial}{\partial \xi^{\prime}}\right)^{\alpha} N\left(x, \xi^{\prime}\right) D^{\alpha} \widetilde{P}(x, \xi) / \alpha ! \\
& =N\left(x, \xi^{\prime}\right) \xi_{0}+\sum_{j, \alpha}\left(\frac{\partial}{\partial \xi}\right)^{\alpha} N_{j}\left(x, \xi^{\prime}\right) D^{\alpha} \Lambda_{k}\left(x, \xi^{\prime}\right) / \alpha !
\end{aligned}
$$

Hence we have

$$
\begin{gathered}
A\left(x, \xi^{\prime}\right) N_{p}\left(x, \xi^{\prime}\right)+P(x, D) N_{p-1}\left(x, \xi^{\prime}\right) \Lambda_{0}\left(x, \xi^{\prime}\right), \\
=\sum_{j+k+|\alpha|=p}\left(\frac{\partial}{\partial \xi}\right)^{\alpha} N_{j}\left(x, \xi^{\prime}\right) D_{x}^{\alpha} \Lambda_{k}\left(x, \xi^{\prime}\right) / \alpha !
\end{gathered}
$$

for $p=0,1, \cdots$. For $p=0$, we have

$$
A\left(x, \xi^{\prime}\right) N_{0}\left(x, \xi^{\prime}\right)=N_{0}\left(x, \xi^{\prime}\right) \Lambda_{0}(x, \xi)
$$

where $N_{0}\left(x, \xi^{\prime}\right)$ is given in Lemma 2.1. Set

$$
\begin{aligned}
F_{p}\left(x, \xi^{\prime}\right)= & \sum_{j+|\alpha|+k=p}\left(\frac{\partial}{\partial \xi}\right)^{\alpha} N_{j}\left(x, \xi^{\prime}\right) D_{x}^{\alpha} \Lambda_{k}\left(x, \xi^{\prime}\right) / \alpha ! \\
& -P(x, D) N_{p-1}\left(x, \xi^{\prime}\right) .
\end{aligned}
$$

Then for $p \geqq 1$, we have from (2.3),

$$
\begin{gathered}
A\left(x, \xi^{\prime}\right) N_{p}\left(x, \xi^{\prime}\right)-N_{p}\left(x, \xi^{\prime}\right) \Lambda_{0}\left(x, \xi^{\prime}\right) \\
=F_{p}\left(x, \xi^{\prime}\right)+N_{0}\left(x, \xi^{\prime}\right) \Lambda_{p}\left(x, \xi^{\prime}\right) .
\end{gathered}
$$

Set

$$
\widetilde{N}_{p}=N_{0}^{-1} N_{p}, \quad \widetilde{F}_{p}=N_{0}^{-1} F_{p},
$$

and 


$$
\begin{aligned}
\widetilde{N}_{p}=\left(\begin{array}{lll}
N_{p}^{(11)} & \cdots & N_{p}^{(1 r)} \\
& \cdots & \\
& \cdots & \\
N_{p}^{(r 1)} & \cdots & N_{p}^{(r r)}
\end{array}\right), & \widetilde{F}_{p}=\left(\begin{array}{lll}
F_{p}^{(11)} & \cdots & N_{p}^{(1 r)} \\
& \cdots & \\
F_{p}^{(r 1)} & \cdots & F_{p}^{(r r)}
\end{array}\right) \\
\Lambda_{p}=\left(\begin{array}{ccc}
\Lambda_{p}^{(1)} & 0 \\
& \ddots & \\
0 & & \Lambda_{p}^{(r)}
\end{array}\right), & \Lambda_{p}^{(l)}=\left(\begin{array}{ccc}
0 & \cdots & 0 \\
0 & \cdots & 0 \\
\gamma_{p 1}^{(l)} & \cdots & \gamma_{p \nu_{l}}^{(l)}
\end{array}\right), \quad(p \geq 1),
\end{aligned}
$$

where $N_{p}^{(i j)}$ and $F_{p}^{(i j)}$ are $\nu_{i} \times \nu_{j}$ matrices. Then we can write from (2.4) for $p \geq 1$,

$$
\begin{aligned}
& \Lambda_{0}^{(i)} N_{p}^{(i i)}-N_{p}^{(i i)} \Lambda_{0}^{(i)}=F_{p}^{(i i)}+\Lambda_{p}^{(i)} \\
& \Lambda_{0}^{(i)} N_{p}^{(i j)}-N_{p}^{(i j)} \Lambda_{0}^{(j)}=F_{p}^{(i j)}, \quad(i \neq j),
\end{aligned}
$$

where

$$
\Lambda_{0}^{(i)}=\lambda^{(i)} I+C^{(\imath)}\left|\xi^{\prime}\right| \text {. }
$$

For (2.6), we can solve $N_{p}^{(i j)}$ as follows

$$
N_{p}^{(i j)}=\sum_{l=0}^{\nu_{j}-1}\left(\lambda^{(i)}-\lambda^{(j)}+C^{(i)}\left|\xi^{\prime}\right|\right)^{-l-1} F_{p}^{(i j)}\left(C^{(j)}\left|\xi^{\prime}\right|\right)^{l}
$$

for $i \neq j, p \geq 1$. We can also solve (2.5), if we choose $\Lambda_{p}^{(i)}$ suitably.

Lemma 2. 3. Let $N$ and $F$ be $m \times m$ matrices and let $C$ be a Jordan's matrix of the form,

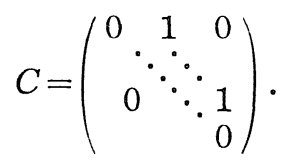

We consider the following linear equations,

$$
C N-N C=F .
$$

Then we can seek for a solution $N$ of (2.7), if and only if the elements $\left\{f_{i, j}\right\}$ of $F$ satisfy,

$$
\sum_{l=0}^{k} f_{m-l, k+1-l}=0, \quad k=0,1, \cdots, m-1 .
$$

Proof. It follows from (2.7) that the elements $\left\{n_{i, j}\right\}$ of $N$ satisfy 


$$
\begin{aligned}
n_{j+1,1} & =f_{j, 1}, \quad j=1, \cdots, m-1, \\
n_{j+1,1} & =f_{j, 1}, \quad j=1, \cdots, m-1, \\
n_{j+1, k} & -n_{j, k-1}=f_{j, k}, \quad k=2, \cdots, m, j=1, \cdots, m-1, \\
-n_{m, k} & =f_{m, k+1}, \quad k=1, \cdots, m-1, \\
0 & =f_{m, 1} .
\end{aligned}
$$

Hence we have from (2.10),

$$
\begin{aligned}
& n_{j+1, k}=\sum_{l=0}^{\min (j, k)-1} f_{j-l, k-l}, \\
& j=1, \cdots, m-1, \quad k=2, \cdots, m .
\end{aligned}
$$

In particular,

$$
n_{m, k}=\sum_{l=1}^{k} f_{m-l, k+1-l}, \quad k=2, \cdots, m
$$

On the other hand, $n_{m, k}$ satisfy (2.11). Hence we have the relation (2.7). We can choose $n_{1, k},(k=1, \cdots, m)$ arbitrarily.

In order to apply this lemma to (2.5), we put

$$
\gamma_{p, k}^{(i)}=-\sum_{l=1}^{k}\left(F_{p}^{(i i)}\right)_{\nu_{i}-k+l, l}
$$

for $k=1, \cdots, \nu_{i}, p=1,2, \cdots$, where $\left(F_{p}^{(i i)}\right)_{s, t}$ stands for the $(s, t)$ element of $F_{p}^{(i i)}$. Then $F_{p}^{(i i)}+\Lambda_{p}^{(i)}$ satisfies (2.7). Hence we can solve (2.5).

Proposition 2. 4. Let $\widetilde{P}$ be the operator given by (2.2). W'e assume that

$\left(L_{1}\right) \quad$ order $\gamma_{k}^{(l)}\left(x, D^{\prime}\right) \leq k-\nu_{l}, \quad k=1, \cdots, \nu_{l}-1, l=1, \cdots, r$.

Then there exists $Q$, a cofactor system of $P$ such that $P Q$ satisfies the condition $(L)$ for $\left(x, \varphi_{x^{\prime}}^{(l)}\right) \in V\left(\widehat{x}, \hat{\xi}^{\prime}\right)$.

Proof. Set

$$
Q=\left(\begin{array}{lll}
Q^{(1)} & 0 \\
& \ddots & \\
0 & & Q^{(r)}
\end{array}\right),
$$


here

$$
\begin{aligned}
& Q^{(l)}=\prod_{k \neq l} q^{(k)}(x, D)^{\nu_{k}} \sum_{k=1}^{\nu_{l}-1} q^{(l)}(x, D)^{\nu_{l}-k}\left(-C^{(l)}\left|D^{\prime}\right|\right)^{k-1}, \\
& q^{(l)}(x, \xi)=\xi_{0}+\lambda^{(l)}\left(x, \xi^{\prime}\right),
\end{aligned}
$$

and set

$$
\begin{aligned}
n_{s}^{(l)} & =s, \quad s=\left\{\sum_{k=1}^{l=1} \nu_{k}\right\}+1, \cdots, \sum_{k=1}^{l} \nu_{k}, \\
& =0, \quad \text { otherwise }
\end{aligned}
$$

Then noting that the condition $\left(\mathrm{L}_{1}\right)$ implies

$$
e^{-i \rho \varphi(l)} \sum_{k=1}^{\nu_{l}-1} \gamma_{k}^{(l)}\left(x, D^{\prime}\right) q^{(l)}(x, D)^{k-1}\left(-C^{(l)}\left|D^{\prime}\right|\right)^{\nu_{l}-k}\left(e^{i \rho \varphi(l)} w\right)=0(1),
$$

for $\left(x, \varphi_{x^{\prime}}^{(l)}\right) \in V\left(\widehat{x}, \hat{\xi}^{\prime}\right)$, we can verify easily that $\widetilde{P} Q$ satisfies the condition (L).

Thus applying Theorem 1.1, we can construct a parametrix of the Cauchy problem for $P$ in some neighborhood $G(\widehat{x})$ of $\widehat{x}$, which implies the existence of the solution of the Cauchy problem (1) for $P$ in $G(x)$, (c.f. [1]). Concerning with the local uniqueness, we must prove that the condition $\left(\mathrm{L}_{1}\right)$ is satisfied for $P^{(*)}$, the adjoint operator of $P$, and that $\left(\mathrm{L}_{1}\right)$ is invariant under the transform of coordinate variables. To do so, we shall prove that our condition $\left(\mathrm{L}_{1}\right)$ is equivalent to the condition $\left(\mathrm{L}_{2}\right)$, given by Petkov. In [7] he has proved that $P^{(*)}$ satisfies $\left(\mathrm{L}_{2}\right)$, if $P$ does so, and that $\left(\mathrm{L}_{2}\right)$ is invariant under the transform of coordinate variables.

We need the following preliminary. The proof is easy.

Proposition 2.5. Let $T\left(x, D^{\prime}\right)$ be an elliptic operator in $L^{0}(G)$ and $S\left(x, D^{\prime}\right)$ be the inverse of $T\left(x, D^{\prime}\right)$, that is, $S\left(x, D^{\prime}\right) T\left(x, D^{\prime}\right)$ $\equiv I, \bmod L^{-\infty}(G)$. If $P$ satisfies $\left(L_{2}\right)$, then $S P T$ does so.

It follows from this proposition that $\widetilde{P}$ given by (2.2) satisfies $\left(\mathrm{L}_{2}\right)$ blookwisely, that is, for any scalar function $f(x) \in C_{0}^{\infty}(G(\widehat{x}))$ and for $\left(x, \varphi_{x^{\prime}}^{(l)}\right) \in V\left(\widehat{x}, \hat{\xi}^{\prime}\right)$, there exist $\nu_{l}$-vector valued functions $v_{k}^{(l)}\left(x, \varphi^{(l)}, f\right)$ $\in C^{\infty}(G(\hat{x}))$ such that 
$\left(\tilde{\mathrm{L}}_{2}\right)$

$$
\begin{gathered}
e^{-i \rho \varphi(l)} \widetilde{P}^{(l)}\left[\left\{f(x) e_{1}^{(l)}+\sum_{k=1}^{\nu_{l}-1} v_{k}^{(l)}\left(x, \varphi^{(l)}, f\right) \rho^{-k}\right\} e^{i \rho \varphi(l)}\right] \\
=0\left(\rho^{-\nu_{l}+1}\right), \quad(\rho \rightarrow \infty), l=1, \cdots, r,
\end{gathered}
$$

where $e_{k}^{(l)}$ is a $\nu_{l}$-vector of which $k$ th-component is 1 and otherwise zero.

Proposition 2. 6. The condition $\left(L_{1}\right)$ is equivalent to $\left(\widetilde{L}_{2}\right)$.

Proof. We can expand asymptotically

$$
\gamma_{k}^{(l)}\left(x, \xi^{\prime}\right)=\sum_{p=1}^{\infty} \gamma_{k p}^{(l)}\left(x, \xi^{\prime}\right),
$$

where functions $\gamma_{k p}^{(l)}\left(x, \xi^{\prime}\right)$ are homogeneous degree $1-p$ in $\xi^{\prime}$. Hence $\left(\mathrm{L}_{1}\right)$ is equivalent to

$$
\gamma_{k p}^{(l)}\left(x, \xi^{\prime}\right)=0, \quad p=1, \cdots, \nu_{l}-k, \quad k=1, \cdots, \nu_{l}-1 .
$$

Therefore it suffices to prove that $\left(\tilde{\mathrm{L}}_{1}\right)$ is equivalent to $\left(\tilde{\mathrm{L}}_{2}\right)$. We write asymptotically

$$
\widetilde{P}^{(l)}=\sum_{j=0}^{\infty} \widetilde{P}_{j}^{(l)}
$$

Then we have

$$
\begin{aligned}
& e^{-\rho \varphi(l)} \widetilde{P}^{(l)}\left[e^{i \rho \varphi(l)}\left\{f(x) e_{1}^{(l)}+\sum_{k=1}^{\nu_{l}-1} v_{k}^{(l)} \rho^{-k}\right\}\right] \\
& =\sum_{p=0}^{\infty} \rho^{1-p}\left\{\sum_{j+k=p} \sigma_{j}\left(\varphi^{(l)}, \widetilde{P}_{k}^{(l)}\right) f(x) e_{1}^{(l)}+\sum_{j+k+s=p} \sigma_{j}\left(\varphi^{(l)}, \widetilde{P}_{k}^{(l)}\right) v_{s}^{(l)}\right\} .
\end{aligned}
$$

Hence $\left(\tilde{\mathrm{L}}_{2}\right)$ is equivalent to

$(2.13)_{p} \quad \sum_{j+k=p} \sigma_{j}\left(\varphi^{(l)}, \widetilde{P}_{k}^{(l)}\right) f(x) e_{1}^{(1)}+\sum_{j+k+s=p} \sigma_{j}\left(\varphi^{(l)}, \widetilde{P}_{k}^{(l)}\right) v_{s}^{(l)}=0$,

for $p=1, \cdots, \nu_{l}-1$. When $p=0$,

$$
\sigma_{0}\left(\varphi^{(l)}, \widetilde{P}_{0}^{(l)}\right) e_{1}^{(l)} f=C^{(l)}\left|\varphi_{x^{\prime}}^{(l)}\right| e_{1}^{(l)} f=0 .
$$

Put $h^{(l)}(x)=\left|\varphi_{x^{\prime}}^{(l)}\right|$. For $p=1$, we have

$$
h^{(l)} C^{(l)} v_{1}^{(l)}+\left(\sigma_{0}\left(\varphi^{(l)}, \widetilde{P}_{1}^{(l)}\right)+\sigma_{1}\left(\varphi^{(l)}, \widetilde{P}_{0}^{(l)}\right)\right) e_{1}^{(l)} f=0 .
$$

Hence there exists $v_{1}^{(l)}$ if and only if

$$
{ }^{t} e_{\nu_{l}}^{(l)}\left\{\sigma_{0}\left(\varphi^{(l)}, \widetilde{P}_{1}^{(l)}\right)+\sigma_{1}\left(\varphi^{(l)}, \widetilde{P}_{0}^{(l)}\right\} e_{1}^{(l)} f=\gamma_{11}^{(l)}\left(x, \varphi_{x^{\prime}}^{(l)}\right) f=0,\right.
$$


which implies

$$
\gamma_{11}^{(l)}\left(x, \xi^{\prime}\right)=0
$$

Then we can find $v_{1}^{(l)}$ of the form

$$
v_{1}^{(l)}=-\frac{1}{h^{(l)}(x)} H_{l}(x, D) f(x) e_{2}^{(l)},
$$

noting,

$$
\sigma_{1}\left(\varphi^{(l)}, \widetilde{P}_{0}^{(l)}\right)=H_{l}(x, D)+\frac{1}{h^{(l)}} C^{(l)}\left\langle\varphi_{x^{\prime}}^{(l)}, D^{\prime}\right\rangle,
$$

where $H_{l}(x, D)=D_{0}+\sum_{j=1}^{n} \lambda_{\xi_{j}}^{(l)}\left(x, \varphi_{x^{\prime}}^{(l)}\right) D_{j}$.

In general we shall prove our statement by induction. Assume that there exist $v_{q}^{(l)}$ satisfying $(2.13)_{q}, q=1, \cdots, p-1$, of the form

$$
v_{q}^{(l)}=\sum_{s=2}^{q+1} a_{s}^{(q)}(x, D) f(x) e_{s}^{(l)}, \quad q=1, \cdots, p-1,
$$

here,

$$
a_{q+1}^{(q)}(x, D)=\left(-\frac{1}{h^{(l)}} H_{l}(x, D)\right)^{q}
$$

if and only if

$$
\gamma_{s q}^{(l)}\left(x, \varphi_{x^{\prime}}^{(l)}\right)=0 \quad \text { for } \quad s+q \leq p
$$

Then we shall prove that we can find $v_{p}^{(l)}$ satisfying $(2.13)_{p}$ of the form $(2.14)_{p+1}$, if and only if $(2.16)_{p+1}$ holds. We have from (2.13) $p$ and $(2.14)_{q}, q \leq p-1$,

$$
\begin{aligned}
h^{(l)} & C^{(l)} v_{p}^{(l)}+\sum_{\substack{j+k+q=p \\
q \leq p-1}} \sigma_{j}\left(\varphi^{(l)}, \widetilde{P}_{k}^{(l)}\right) v_{q}^{(l)}+\sum_{j+k=p} \sigma_{j}\left(\varphi^{(l)}, \widetilde{P}_{k}^{(l)}\right) e_{1}^{(l)} f \\
= & h^{(l)} C^{(l)} v_{p}^{(l)}+\sum_{\substack{j+k+q=p \\
q \leq p-1}} \sum_{s=1}^{q+1} \sigma_{j}\left(\varphi^{(l)}, \widetilde{P}_{k}^{(l)}\right) a_{s}^{(q)} f e_{s}^{(l)} \\
& +\sum_{j=k} \sigma_{j}\left(\varphi^{(l)}, \widetilde{P}_{k}^{(l)}\right) f e_{1}^{(l)}=0 .
\end{aligned}
$$

Hence we can find $v_{p}^{(l)}$ if and only if

$$
{ }^{t} \boldsymbol{e}_{\nu_{l}}^{(l)}\left\{\sum_{\substack{j+\underset{k+q=p}{q \leq p-1} \\ q \leq p-1}} \sigma_{j}\left(\varphi^{(l)}, \widetilde{P}_{k}^{(l)}\right) \sum_{s=2}^{q+1} a_{s}^{(q)}(x, D) f \boldsymbol{e}_{s}^{(l)}\right.
$$




$$
\left.+\sum_{j+k=p} \sigma_{j}\left(\varphi^{(l)}, \widetilde{P}_{k}^{(l)}\right) f e_{1}^{(l)}\right\}=0 .
$$

It follows from $(2.16)_{p}$ that

$$
{ }^{t} e_{\nu l}^{(l)} \sigma_{j}\left(\varphi^{(l)}, \widetilde{P}_{k}^{(l)}\right) e_{s}^{(l)}=\sigma_{j}\left(\varphi^{(l)}, \gamma_{s k}^{(l)}\right)=0, \quad s+k \leq p,
$$

for any $j$. Hence we have from (2.18)

$$
\begin{aligned}
& \sum_{\substack{j+k+q=p \\
q \leq p-1}} \sigma_{j}\left(\varphi^{(l)}, \gamma_{s k}^{(l)}\right) \sum_{s+k=p+1} a_{s}^{(q)}(x, D) f+\sigma_{0}\left(\varphi^{(l)}, \gamma_{1 p}^{(l)}\right) f \\
& =\sum_{k+q=p} \sigma_{0}\left(\varphi^{(l)}, \gamma_{q+1}^{(l)}\right) a_{q+1}^{(q)}(x, D) f+\sigma_{0}\left(\varphi^{(l)}, \gamma_{1 p}^{(l)}\right) f=0 .
\end{aligned}
$$

Since $\left\{1, a_{q+1}^{(q)}(x, D), q=1, \cdots, p-1\right\}$ is linearly independent from (2.15) ${ }_{q}$, $q \leq p-1$, we obtain $(2.16)_{p+1}$. Inserting $(2.14)_{p}$ into (2.17), we obtain by virtue of $(2.16)_{p+1}$,

$$
\left.\sum_{j+q=p} \sigma_{j}\left(\varphi^{(l)}, \widetilde{P}_{0}^{(l)}\right) a_{s}^{(q)}(x, D) f e_{s}^{(l)}+\sigma_{p}\left(\varphi^{(l)}\right), \widetilde{P}_{0}^{(l)}\right) f e_{1}^{(l)}=0
$$

Hence, noting that

$$
\sigma_{j}\left(\varphi^{(l)}, \widetilde{P}_{0}^{(l)}\right)=I \sigma_{j}\left(\varphi^{(l)}, q^{(l)}\right)+\sigma_{j}\left(\varphi^{(l)},\left|D^{\prime}\right|\right) C^{(l)}
$$

we obtain from (2.19)

$$
a_{q+1}^{(p)}(x, D)=-\frac{1}{h^{(l)}}\left\{\sum_{s=q-1}^{p-1} \sigma_{p-s}\left(\varphi^{(l)}, q^{(l)}\right) a_{q}^{(s)}+\sum_{s=q}^{p-1} \sigma_{p-s}\left(\varphi^{(l)},\left|D^{\prime}\right|\right) a_{q+1}^{(s)}\right\}
$$

for $q=1, \cdots, p$, where $a_{1}^{(0)}=1$. In particular for $q=p$, we obtain $(2.15)_{p}$.

\section{$\S 3$. Proof of the Necessity Part of Theorem 3}

In this section we shall show that the condition $\left(\mathrm{L}_{1}\right)$ is necessary in order that the Cauchy problem for $P$ is well posed. Assume that the Cauchy problem for $P$ is well posed at $\hat{x} \in G$. Then for any neighborhood $U(\hat{x})$ of $\hat{x}$, there exists a neighborhood $G(\hat{x}) \subset U(\widehat{x})$ and a positive integer $s_{0}$ such that

$$
|u|_{0, G^{+}(\hat{x})} \leq C(x)\left\{\left|P u_{s_{0}, G^{+}(\hat{x})}+\right| u_{\left.\left.\right|_{s_{0}, G_{0}(\hat{x})}\right\}}\right.
$$

for $u \in C^{\infty}(U(\widehat{x}))$, where $G^{+}(\widehat{x})=\left\{x \in G(\widehat{x}), x_{0}>\widehat{x}_{0}\right\}$ and $G_{0}(\widehat{x})=\{x$ $\left.\in G(\hat{x}), x_{0}=\widehat{x}_{0}\right\}$. This inequality is derived by the closed graph theorem. We shall prove the necessity of Theorem 3 by contradiction, that is, we shall construct an asymptotic solution which does not satisfy the 
inequality (3.1).

By virtue of Proposition 2.2 we can transform $P$ to $\widetilde{P}$ by $N$, where $\widetilde{P}$ has the form (2.2). Then we assume that the property $\left(\mathrm{L}_{1}\right)$ is not valid for some $l_{0}$ and some $k_{0}$. We introduce a cofactor system $Q^{\left(l_{0}\right)}$ of $P^{\left(l_{0}\right)}$ such that

$$
Q^{\left(l_{0}\right)}=\sum_{k=0}^{\nu_{l_{0}-1}} q^{\left(l_{0}\right)}(x, D)^{\nu_{l_{0}-1-k}}\left(-C^{\left(l_{0}\right)}\left|D^{\prime}\right|\right)^{k},
$$

where $q^{\left(l_{0}\right)}(x, D)=D_{0}+\lambda^{\left(l_{0}\right)}\left(x, D^{\prime}\right)$. Then we have

$$
P^{\left(l_{0}\right)}(x, D) Q^{\left(l_{0}\right)}(x, D)=I_{\nu l_{0}}\left(q^{\left(l_{0}\right)}(x, D)\right)^{\nu l_{0}}+\left\{b_{t}^{s}(x, D)\right\}_{s, t=1, \cdots, \nu l_{0}},
$$

where

$$
\begin{gathered}
b_{t}^{s}(x, D)=0, \quad t \leq s \leq \nu_{l_{0}}-1, \\
b_{s+k}^{s}(x, D)=\left[q^{\left(l_{0}\right)}(x, D)^{\nu_{l_{0}}-k},\left|D^{\prime}\right|\right]\left(-\left|D^{\prime}\right|\right)^{k-1}, \\
s=1, \cdots, \nu_{l_{0}}-k, \quad k=1, \cdots, \nu_{l_{0}}-1, \\
b_{t}^{\nu_{l_{0}}}(x, D)=\sum_{k=1}^{t} \gamma_{k}^{\left(l_{0}\right)}\left(x, D^{\prime}\right) q^{\left(l_{0}\right)}(x, D)^{k-1}\left(-\left|D^{\prime}\right|\right)^{\nu_{l_{0}}-1-t+k}, \\
t=1, \cdots, \nu_{l_{0}} .
\end{gathered}
$$

We shall construct the asymptotic solutions of the following equations (put $\nu_{l_{0}}=\nu$ for simplicity),

$$
q^{\left(l_{0}\right)}(x, D){ }^{\nu} v^{s}+\sum_{t=1}^{\nu} b_{t}^{s}(x, D) v^{t}=0 \quad s=1, \cdots, \nu .
$$

We seek $v^{s}=v^{s}(x, \rho)$ of the form,

$$
\begin{aligned}
& v^{s}(x, \rho)=\rho^{-n_{s}} e^{i E(\rho, x)} \sum_{j=0}^{\infty} \rho^{-j \varepsilon_{v}} v_{j}^{s}(x), \\
& E(\rho, x)=\sum_{i=0}^{d} \rho^{\sigma_{i}} \varphi^{(i)}(x), \\
& 1=\sigma^{(0)}>\sigma^{(1)}>\cdots>\sigma^{(d)}>0,
\end{aligned}
$$

and $\varepsilon^{-1}$ is the common denominator of the rational numbers $\sigma^{(j)} \quad(j=1$, $\cdots, d)$. We shall determine $\left(\sigma^{(j)}, \varphi^{(j)}\right)$ inductively.

At first we define $\varphi^{(0)}$ as follows

$$
\begin{aligned}
& \varphi_{x_{0}}^{(0)}+\lambda^{\left(l_{0}\right)}\left(x, \varphi_{x^{\prime}}^{(0)}\right)=0 \\
& \left.\varphi^{(0)}\right|_{x_{0}=\hat{x}_{0}}=\left\langle x^{\prime}, \omega^{(0)}\right\rangle, w^{(0)} \in \mathbb{R}^{n} \backslash 0 .
\end{aligned}
$$


We put

$$
\begin{aligned}
L & =q^{\left(l_{0}\right)}(x, D)^{\nu}+b_{\nu}^{\nu}(x, D) \\
& =\sum_{k=0}^{\nu} L_{k}\left(x, D^{\prime}\right) q^{\left(l_{0}\right)}(x, D)^{k-1},
\end{aligned}
$$

where $L_{0}=1$ and $L_{k}$ are pseudo-differential operators in $x^{\prime}$. We denote by $d_{k}^{(0)}$ the order of $L_{k}$. Then from (3.2) we have

$$
\nu_{l_{0}}-k \geq d_{k}^{(0)} \geq \operatorname{order} \gamma_{k}^{\left(l_{0}\right)}+\nu_{l_{0}}-k, k=1, \cdots, \nu_{l_{0}} .
$$

We define

$$
\begin{aligned}
L_{\rho}^{(0)} w & =e^{-i \rho \varphi(0)} L\left(e^{i \rho \varphi(0)} w\right) \\
& =\sum_{l \geq 0} \rho^{d_{k}^{(0)-l}} \sigma_{l}\left(L_{k}, \varphi^{(0)}\right) \sum_{j \geq 0} \rho^{-k} \sigma_{k-1+j}\left(q^{\left(l_{0}\right)(k-1)}, \varphi^{(0)}\right) \\
& =\sum_{k=0}^{\nu} \sum_{s \geq 0} \rho^{d_{k}^{(0)-s}} L_{k, s}^{(0)}(x, D) w,
\end{aligned}
$$

where

$$
L_{k, 8}^{(0)}=\sum_{l+j=s} \sigma_{l}\left(L_{k}, \varphi^{(0)}\right) \sigma_{k-1+j}\left(q^{\left(l_{0}\right)(k-1)}, \varphi^{(0)}\right) .
$$

Then from Lemma 1.2 it follows that the principal part of $\sigma_{k-1}\left(q^{\left(l_{0}\right)(k-1)}, \varphi^{(0)}\right)$ is given by

$$
H(x, \hat{\xi})^{(k-1)}
$$

where $H(x, \xi)=\xi_{0}+\sum_{i=j}^{n} \lambda_{\xi_{i}}^{\left(l_{0}\right)}\left(x, \varphi_{x^{\prime}}^{(0)}\right) \xi_{i}$. We note that the order of $L_{k, s}^{(0)} \leq$ $k-1+s$. We put

$$
\begin{aligned}
& \sigma^{(1)}=\max _{1 \leq k \leq \nu-1} \frac{d_{k}^{(0)}}{\nu-k+1}, \\
& \psi^{(1)}=\left\{k, \frac{d_{k}^{(0)}}{\nu-k+1}=\sigma^{(1)}\right\} .
\end{aligned}
$$

Then if $\left(L_{1}\right)$ is not valid, we have from (3.5)

$$
d_{k}^{(0)}>0
$$

for some $k$. Therefore we have

$$
0<\sigma^{(1)}<1
$$

We define 


$$
\begin{aligned}
L_{\rho}^{(1)} & =\exp \left\{-i \rho^{\sigma(1)} \varphi^{(1)}\right\} L_{\rho}^{(0)} \exp \left\{i \rho^{\sigma(1)} \varphi^{(1)}\right\} \\
& =\sum \rho^{d_{k^{(0)}-s+\sigma(1)(k-1+s-j)}} \sigma_{j}\left(L_{k, 0}^{(0)}, \varphi^{(1)}\right) \\
& =\rho^{\sigma^{(1)} \nu}\left\{\left(H(x, D) \varphi^{(1)}\right)^{\nu}+\sum_{k \in \#(1)} \sigma_{0}\left(L_{k, 0}^{(0)}, \varphi^{(1)}\right)+o(1)\right\} .
\end{aligned}
$$

We put

$$
h^{(1)}\left(x, \varphi_{x}^{(1)}\right)=H\left(x, \varphi_{x}^{(1)}\right)^{\nu}+\sum_{k \in \#(1)} \sigma_{0}\left(L_{k, 0}^{(0)}, \varphi^{(1)}\right),
$$

which is a polynomial in $\varphi_{x}^{(1)}$. But from (3.6) and (3.7) it follows that the principal part of $L_{k, 0}^{(0)}$ is given by $\sigma_{0}\left(L_{k}, \varphi^{(0)}\right) H(x, \xi)^{\nu-k}$. Hence $h^{(1)}\left(x, \varphi_{x}^{(1)}\right)$ is a polynomial in $H\left(x, \varphi_{x}^{(1)}\right)$ and is decomposed

$$
h^{(1)}\left(x, \varphi_{x}^{(1)}\right)=\left(H\left(x, \varphi_{x}^{(1)}\right)-C^{(1)}(x)\right)^{m(1)} Q^{(1)}(x, H)
$$

for $x \in U^{(1)}$, an open set, where $Q^{(1)}(x, H)$ is a polynomial in $H$, $Q^{(1)}\left(x, C^{(1)}\right) \neq 0$ and $C^{(1)}(x)$ is a $C^{\infty}$-function in $U^{(1)}$. We note that we can choose $C^{(1)}(x)$ such that

$$
\operatorname{Im} C^{(1)}(x)<0 \text { in } U^{(1)} .
$$

For, we have

$$
\begin{aligned}
h^{(1)}\left(x, \varphi_{x}^{(1)}\right) & =H\left(x, \varphi_{x}^{(1)}\right)^{\nu}+\sum_{k \in \#(1)} \hat{L}\left(x, \varphi_{x}^{(0)}\right) H\left(x, \varphi_{x}^{(1)}\right)^{\nu-k} \\
& =\widehat{h}^{(1)}\left(x, H, \varphi_{x}^{(0)}\right)
\end{aligned}
$$

where $\widehat{L}_{k}\left(x, \varphi_{x}^{(0)}\right)$, the principal part $L_{k}$, is a polynomial of order $\sigma^{(1)} k$ for $k \in w^{(1)}$. Hence we have

$$
\begin{aligned}
\widehat{h}^{(1)}\left(x, H,-\varphi_{x}^{(0)}\right)= & H^{\nu}+\sum_{k \in \#(1)}(-1)^{\sigma(1) k} L_{k}\left(x, \varphi_{x}^{(0)}\right) H^{\nu-k} \\
& =(-1)^{\nu \sigma(1)} \widehat{h}^{(1)}\left(x,(-1)^{\sigma(1)} H, \varphi_{x}^{(0)}\right) .
\end{aligned}
$$

Therefore $(-1)^{-\sigma^{(1)}} C^{(1)}(x)$ is a root of $h^{(1)}\left(x, H,-\varphi_{x}^{(0)}\right)=0$. Since $C^{(1)}(x) \neq 0$ and $0<\sigma^{(1)}<1$, we can choose a branch of $(-1)^{\sigma(1)}$ such that $\operatorname{Im}(-1)^{-\sigma(1)} C^{(1)}(x)<0$.

We choose $\varphi^{(1)}$ as a solution

$$
\left\{\begin{array}{l}
H\left(x, \varphi_{x}^{(1)}\right)=C^{(1)}(x) \\
\left.\varphi^{(1)}\right|_{x_{0}=\hat{x}_{0}}=\left\langle x^{\prime}, \omega^{(1)}\right\rangle, \omega^{(1)} \in R^{n} \backslash 0 .
\end{array}\right.
$$

Then (3.9) implies 


$$
\operatorname{Im} \varphi^{(1)}<0, \quad x \in U^{(1)}, \quad x_{0}>\widehat{x}_{0} .
$$

We define $L^{(j)}, \varphi^{(j)}, \sigma^{(j)}$ and $h^{(j)}(x, H)$ inductively, for $j \geq 2$,

$$
\begin{aligned}
& L_{\rho}^{(j)}=\exp \left\{-i \rho^{\sigma^{(j)}} \varphi^{(j)}\right\} L_{\rho}^{(j-1)} \exp \left\{i \rho^{\sigma^{(j)}} \varphi^{(j)}\right\}, \\
& =\rho^{M^{(j)}} \sum_{k \geq 0} \rho^{-k \xi(j)} L_{k}^{(j)}, \\
& L_{0}^{(j)}=h^{(j)}\left(x, H\left(x, \varphi_{x}^{(j)}\right)\right) \\
& =\left(H\left(x, \varphi_{x}^{(j)}\right)-C^{(j)}(x)\right)^{m^{(j)}} Q^{(j)}(x, H) \quad \text { in } U^{(j)} \subset U^{(j-1)} \text {, } \\
& d_{k}^{(j)}=\operatorname{order} L_{k}^{(j)}, \quad\left(d_{k}^{(j)}=-\infty, \quad \text { if } \quad L_{k}^{(j)} \equiv 0\right), \\
& \sigma^{(j)}=\max _{0<k<m^{(j-1)} \sigma^{(j-1) / \varepsilon^{(j-1)}}} \frac{m^{(j-1)} \sigma^{(j-1)}-k \varepsilon^{(j-1)}}{m^{(j-1)}-d_{k}^{(j-1)}},
\end{aligned}
$$

$\varepsilon^{(j)-1}$; the common denominator of the rational numbers $\sigma^{(1)}, \cdots, \sigma^{(j)}$,

$$
\begin{aligned}
&=^{(j)}=\left\{0<k<m^{(j-1)} \sigma^{(j-1)} / \varepsilon^{(j-1)}, \frac{m^{(j-1)} \sigma^{(j-1)}-k \varepsilon^{(j-1)}}{m^{(j-1)}-d_{k}^{(j-1)}}=\sigma^{(j)}\right\} \cup\{0\}, \\
& M^{(j)}=M^{(j-1)}+m^{(j-1)}\left(\sigma^{(j)}-\sigma^{(j-1)}\right), \quad M^{(1)}=\nu \sigma^{(1)}, \\
& \nu>m^{(1)}>\cdots>m^{(j)}, \quad 1>\sigma^{(1)}>\cdots>\sigma^{(j)},
\end{aligned}
$$

and $\varphi^{(j)}$ a solution as

$$
\begin{aligned}
& H\left(x, \varphi_{x}^{(j)}\right)=C^{(j)}(x) \\
& \left.\varphi^{(j)}\right|_{x_{0}=\hat{x}_{0}}=\left\langle x^{\prime}, \omega^{(j)}\right\rangle,
\end{aligned}
$$

where $H(x, \xi)=\xi_{0}+\sum_{i=1}^{n} \lambda_{\xi_{i}}^{\left(l_{0}\right)}\left(x, \varphi_{x^{\prime}}^{(0)}\right) \xi_{i}$.

We must prove that $L_{0}^{(j)}$, the coefficient of the leading power $\rho^{M^{(j)}}$ in $L_{p}^{(j)}$ is a polynomial in $H\left(x, \varphi_{x}^{(j)}\right)$. To do so, we decompose $L_{k}^{(j-2)}$ such that

$$
L_{k}^{(j-2)}=\sum_{s=0}^{d_{k}(j-2)} L_{k, s}^{(j-2)}\left(x, D^{\prime}\right) H(x, D)^{d_{k}^{(j-2)-s}}
$$

where $L_{k, s}^{(j-2)}$ is a differential operator in $x^{\prime}$ of order $s$. We rewrite $H(x, D)^{k}$ as

$$
\begin{aligned}
H(x, D)^{k} & =\left(H(x, D)-\rho C^{(j-1)}(x)+\rho C^{(j-1)}(x)\right)^{k} \\
& =\sum_{p=0}^{k} C_{k, p}^{(j-1)}(x) \rho^{p}\left(H-\rho C^{(j-1)}(x)\right)^{k-p},
\end{aligned}
$$


where $C_{k, 0}^{(j-1)}=1$. Then we have

$$
L_{\rho}^{(j-2)}=\rho^{M^{(j-2)}} \sum \rho^{-k \varepsilon^{(j-2)}+p \sigma^{(j-1)}} L_{k, s}^{(j-2)} C_{d_{k}^{(j-1)-s, p}}^{(j-1)}\left(H-\rho^{\sigma^{(j-1)}} C^{(j-1)}\right)^{d_{k-s-p}^{(j-2)}} .
$$

Hence we obtain

$$
\begin{aligned}
L_{\rho}^{(j-1)}= & \exp \left\{-i \rho^{\sigma^{(j-1)}} \varphi^{(j-1)}\right\} L_{\rho}^{(j-2)} \exp \left\{i \rho^{\sigma^{(j-1)}} \varphi^{(j-1)}\right\} \\
= & \rho^{M^{(j-2)}} \sum \rho^{-k \varepsilon^{(j-2)}+(p+s-l) \sigma^{(j-1)}} \sigma_{l}\left(L_{k, s}^{(j-2)}, \varphi^{(j-1)}\right) C_{d_{k}(j-2)-s, p}^{(j-1)}(x) \\
& \times H(x, D)^{d_{k}(j-2)-s-p}, \\
= & \rho^{M^{(j-1)}} \sum_{t} \rho^{-t \varepsilon^{(j-1)}} L_{t}^{(j-1)} .
\end{aligned}
$$

Therefore we have

$$
L_{t}^{(j-1)}=\sum \sigma_{l}\left(L_{k, s}^{(j-2)}, \varphi^{(j-1)}\right) C_{d_{k}(j-2)-s, p}^{(j-1)}(x) H(x, D)^{d_{k}^{(j-2)-s-p}},
$$

where the summation is

$$
k \varepsilon^{(j-2)}-(p+s-l) \sigma^{(j-1)}+m^{(j-2)}\left(\sigma^{(j-1)}-\sigma^{(j-2)}=t \varepsilon^{(j-1)} .\right.
$$

On the other hand, we have

$$
\begin{aligned}
L_{\rho}^{(j)} & =\exp \left\{-i \rho^{\sigma(j)} \varphi^{(j)}\right\} L_{\rho}^{(i-1)} \exp \left\{i \rho^{\sigma(j)} \varphi^{(j)}\right\} \\
& =\rho^{M M^{(j-1)}} \sum \rho^{-t \varepsilon^{(j-1)}+\sigma^{(j)}\left(d_{t}^{(j)}-q\right)} \sigma_{q}\left(L_{t}^{(j-1)}, \varphi^{(j)}\right) \\
& =\rho^{M^{(j)}}\left(\sum_{t \in \#(j)} \sigma_{0}\left(L_{t}^{(j-1)}, \varphi^{(j)}\right)+o(1)\right) .
\end{aligned}
$$

Hence we have

$$
L_{0}^{(j)}=\sum_{t \in \#(j)} \sigma_{0}\left(L_{t}^{(j-1)}, \varphi^{(j)}\right) .
$$

In order to prove that $L_{0}^{(j)}$ is a polynomial in $H\left(x, \varphi_{x}^{(j)}\right)$, it suffices to know that the principal part of $L_{t}^{(j-1)}$ for $t \in \psi^{(j)}$ is a polynomial in $H\left(x, \varphi_{x}^{(j)}\right)$, that is, in the expression of $L_{t}^{(j-1)}$ for $t \in \psi^{(j)}$ the terms $\sigma_{l}\left(L_{k, s}^{(j-1)}, \varphi^{(j-1)}\right)$ become zero for $l>0$. The principal part of $L_{t}^{(j-1)}$ is given by

$$
\sum \hat{\sigma}_{l}\left(L_{k, s}^{(j-2)}, \varphi^{(j-1)}\right) H(x, \xi)^{d_{k}^{(j-2)-s}},
$$

where the summation is

$$
l+d_{k}^{(j-2)}-s=d_{t}^{(j-1)},
$$

for, the order of $L_{t}^{(j-1)}$ is equal to $d_{t}^{(j-1)}$, where $\hat{\sigma}_{l}\left(L_{k, s}^{(j-2)}, \varphi^{(j-1)}\right)$ stands for the principal part of $\sigma_{l}\left(L_{k, s}^{(j-2)}, \varphi^{(j-1)}\right)$. Assume that for some $\hat{l} \neq 0$ 
and $\hat{s}$,

$$
\sum_{i+d_{k, j}^{(j-2)}-\hat{s}=d_{t}^{(j-1)}} \hat{\sigma}_{\hat{l}}\left(L_{k, \hat{s}}^{(j-2)}, \varphi^{(j-1)}\right) \neq 0 .
$$

Since the principal part $\hat{L}_{k, \hat{s}}^{(j-2)}(x, \xi)$ of $L_{k, \hat{s}}^{(j-2)}$ is a homogeneous polynomial in $\xi$ of order $\hat{s}$ and $\hat{\sigma}_{l}\left(L_{k, s}^{(j-2)}, \varphi^{(j-1)}\right)$ is given by

$$
\sum_{|\alpha|=\imath} \frac{1}{\alpha !}\left(\frac{\partial}{\partial \xi^{\prime}}\right)^{\alpha} \widehat{L}_{k, s}^{(j-2)}\left(x, \varphi_{x^{\prime}}^{(j-1)}\right) \xi^{\prime \alpha}
$$

which homogeneous in $\varphi_{x^{\prime}}^{(j-1)}$. Hence if (3.12) is valid,

$$
\sum_{i+d_{k}(j-2)-\hat{s}=d_{t}(j-1)} \hat{\sigma}_{\hat{l}-1}\left(L_{k, \hat{s}}^{(j-2)}, \varphi^{(j-1)}\right)
$$

does not vanish, if we choose a suitable $\omega^{(j-1)}$, the direction of the initial data of $\varphi^{(j-1)}$. On the other hand

$$
\sum_{\hat{l}_{+} d_{k}(j-2)-\hat{s}=d_{t}(j-1)} \hat{\sigma}_{\hat{l}-1}\left(L_{k, \xi}^{(j-2)}, \varphi^{(j-1)}\right) H(x, \xi)^{d_{k}(j-2)-\hat{s}}
$$

is involved in the terms of $L_{t-\sigma^{(j-1) / \varepsilon(j-1)}}^{(j-1)}$. Hence we have

$$
d_{t-\sigma^{(j-1) / \varepsilon^{(j-1)}}}^{(j-1)} \geq d_{t}^{(j-1)}-1
$$

which contradicts to the definition of $\sigma^{(j)}$. For,

$$
\sigma^{(j)}=\frac{m^{(j-1)} \sigma^{(j-1)}-t \varepsilon^{(j-1)}}{m^{(j-1)}-d_{t}^{(j-1)}}
$$

and on the other hand, $\sigma^{(j-1)}>\sigma^{(j)}$ implies

$$
\frac{m^{(j-1)} \sigma^{(j-1)}-\left(t-\sigma^{(j-1)} / \varepsilon^{(j-1)}\right)}{m^{(j-1)}-d_{t-\sigma^{(j-1) / 6}(j-1)}^{(j-1)}}>\frac{m^{(j-1)} \sigma^{(j-1)}-t \varepsilon^{(j-1)}+\sigma^{(j-1)}}{m^{(j-1)}-d_{t}^{(j-1)}+1}>\sigma^{(j)} .
$$

Thus we have proved that $L_{0}^{(j)}$ is a polynomial in $H\left(x, \varphi_{x}^{(j)}\right)$, that is,

$$
h^{(j)}\left(x, H\left(x, \varphi_{x}^{(j)}\right)=L_{0}^{(j)}=\sum_{t \in \#(j)} \sigma_{0}\left(L_{t}^{(j-1)}, \varphi^{(j)}\right) .\right.
$$

Then the coefficient of the leading power $H^{m^{(j-1)}}$ in $L_{0}^{(j)}$ is $Q^{(j-1)}\left(x, C^{(j-1)}\right)$ $\neq 0$. Hence there exists an open set $U^{(j)} \subset U^{(j-1)}$ such that we can decompose

$$
h^{(j)}(x, H)=\left(H-C^{(j)}(x)\right)^{m^{(j)}} Q^{(j)}(x, H) \text { in } U^{(j)},
$$

where $C^{(j)}(x) \in C^{\infty}\left(U^{(j)}\right)$ and $Q^{(j)}\left(x, C^{(j)}\right) \neq 0$. Moreover we note that

$$
m^{(j)}<m^{(j-1)},
$$


if $\sigma^{(j)}>0$, that is, $L_{k}^{(j-1)} \neq 0$ for some $k<m^{(j-1)} \sigma^{(j-1)} / \varepsilon^{(j-1)}$.

Next we shall prove that

$$
d_{k}^{(j)}<k \varepsilon^{(j)} / \sigma^{(j)} \quad \text { for } \quad 0<k<m^{(j)} \sigma^{(j)} / \varepsilon^{(j)} .
$$

We have

$$
\begin{aligned}
L_{\rho}^{(j)} & =\exp \left\{-i \rho^{\sigma^{(j)}} \varphi^{(j)}\right\} L_{\rho}^{(j-1)} \exp \left\{i \rho^{\sigma^{(j)}} \varphi^{(j)}\right. \\
& =\rho^{M^{(j-1)}} \sum \rho^{-\varepsilon^{(j-1)} p+\sigma^{(j)}\left(d_{p}^{(j-1)}-s\right)} \sigma_{s}\left(L_{p}^{(j-1)}, \varphi^{(j)}\right) \\
& =\rho^{M^{(j)}} \sum \rho^{-\varepsilon^{(j)} k} L_{k}^{(j)} .
\end{aligned}
$$

Hence we obtain

$$
L_{k}^{(j)}=\beth \sigma_{s}\left(L_{p}^{(j-1)}, \varphi^{(j)}\right)
$$

where the summation is,

(3. 18) $\quad \varepsilon^{(j-1)} p-\sigma^{(j)}\left(d_{p}^{(j-1)}-s\right)+m^{(j-1)}\left(\sigma^{(j)}-\sigma^{(j-1)}\right)=k \varepsilon^{(j)}$.

If $k \varepsilon^{(j)} / \sigma^{(j)}$ is not an integer, it is evident that (3.18) implies (3.16). When $k \varepsilon^{(j)} / \sigma^{(j)}$ is an integer, it follows from (3.14) that the term of order $k \varepsilon^{(j)} / \sigma^{(j)}$ in $L_{k}^{(j)}$ is given by

$$
\begin{aligned}
\underset{p \in \#(j)}{Y i} \hat{\sigma}_{s} & \left(L_{p}^{(j-1)}, \varphi^{(j)}\right) \\
= & \frac{1}{s !}\left(\frac{\partial}{\partial H}\right)^{s} h^{(j)}\left(x, H\left(x, \varphi_{x}^{(j)}\right)\right)=0
\end{aligned}
$$

for $s=k \varepsilon^{(j)} / \sigma^{(j)}<m^{(j)}$, if $k<m^{(j)} \sigma^{(j)} / \varepsilon^{(j)}$. Thus we have proved (3.16). It is evident that (3.16) implies that $\sigma^{(j+1)}<\sigma^{(j)}$, if $\sigma^{(j)} \neq 0$. Moreover from (3.17) we have

$$
L_{k}^{(j)}=\sum_{s=0}^{m^{(j)}} L_{k, s}^{(j)}(x) H(x, D)^{m^{(j)}-s}
$$

for $k=m^{(j)} \sigma^{(j)} / \varepsilon^{(j)}$, where $L_{k, 0}^{(j)}=Q^{(j)}\left(x, C^{(j)}(x)\right) \neq 0$ for $k=m^{(j)} \sigma^{(j)} / \varepsilon^{(j)}$. Thus it follows from (3.15) that in the finite step we have

$$
L_{p}^{(d)}=\rho^{M I^{(d)}} \sum_{k \geq k(d)} \rho^{-\varepsilon^{(d)} k} L_{k}^{(d)}
$$

where $k(d)=m^{(d)} \sigma^{(d)} / \varepsilon^{(d)}$ and $L_{k(d)}^{(d)}$ is given by (3.19) with $j=d$.

Now we return to construct asymptotic solutions of the equations (3.3). Noting that from (3.2) we have, $\left(n_{s}=\left(1-\sigma^{(1)}\right) s\right)$, 


$$
\begin{gathered}
\rho^{-n_{s}} e^{-i E(\rho, x)} q^{\left(l_{0}\right)}(x, D){ }^{\nu} e^{i E(\rho, x)} \\
=\rho^{\nu \sigma(1)-n_{s}}\left(H\left(x, \varphi_{x}^{(1)}\right)^{\nu}+0\left(\rho^{-\sigma(1)}\right)\right), \\
\rho^{-n_{t}} e^{-i E(\rho, x)} b_{t}^{s}(x, D) e^{i E(\rho, x)}=0\left(\rho^{-n_{s}+\sigma(1)(\nu-1)}\right), \\
s=1, \cdots, \nu-1, t=1, \cdots, \nu, \\
\rho^{-n_{t}} e^{-i E} b_{t}^{\nu}(x, D) e^{i E}=0\left(\rho^{M(d)}-n_{\nu}\right) \\
t=1, \cdots, \nu-1,
\end{gathered}
$$

and inserting $v^{s}(x, \rho)$ into (3.3), we obtain by virtue of (3.20),

$$
\begin{aligned}
& q^{\left(l_{0}\right)}(x, D)^{\nu} v^{s}+\sum_{t=1}^{\nu} b_{t}^{s}(x, D) v^{t} \\
& =e^{i E(\rho, x)} \rho^{\nu \sigma(1)} \sum_{j \geq 0} \rho^{-j \sigma}\left(H\left(x, \varphi_{x}^{(1)}\right)^{\nu} v_{j}^{s}+g_{t}^{s}\right), \\
& \quad s=1, \cdots, \nu-1, \\
& q^{\left(l_{0}\right)}(x, D)^{\nu} v^{\nu}+\sum_{t=1}^{\nu} b_{t}^{\nu}(x, D) v^{t} \\
& =L(x, D) v^{\nu}+\sum_{t=1}^{\nu-1} b_{t}^{\nu}(x, D) v^{t} \\
& =e^{i E(\rho, x)} \rho^{M M^{(d)}} \sum_{j \geq 0} \rho^{-j \sigma}\left(L_{k(d)}^{(d)} v_{j}^{\nu}+g_{j}^{\nu}\right),
\end{aligned}
$$

where we put $\nu=\nu_{l_{0}}$ and $\sigma=\varepsilon^{(d)}$, and $g_{t}^{s}$ are functions of $\left(x, v_{0}^{1}, \cdots\right.$, $\left.v_{j-1}^{1}, \cdots, v_{1}^{\nu}, \cdots, v_{j-1}^{\nu}\right)$ and $g_{j}^{\nu}$ functions of $\left(x, v_{0}^{1}, \cdots, v_{j}^{1}, \cdots, v_{0}^{\nu-1}, \cdots, v_{j}^{\nu-1}\right.$, $\left.v_{0}^{\nu}, \cdots, v_{j-1}^{\nu}\right)$, and in particular $g_{0}^{s}=0, s=1, \cdots, \nu$, and $L_{0}^{(d)}$ is given by (3. 19).

Thus we have the following equations,

$$
\left\{\begin{array}{l}
H\left(x, \varphi_{x}^{(1)}\right)^{\nu} v_{j}^{s}+g_{j}^{s}=0, \quad s=1, \cdots, \nu-1, \\
L_{k(d)}^{(d)}(x, D) v_{j}^{\nu}+g_{j}^{\nu}=0
\end{array}\right.
$$

for $j=0,1,2, \cdots$. Since $H\left(x, \varphi_{x}^{(1)}\right)=C^{(1)}(x) \neq 0$ and $L_{k(d)}^{(d)}$ involved only the differential operator $H(x, D)$, and $g_{0}=0$, we can solve (3.21) successively. Since $g_{0}^{s}=0, s=1, \cdots, \nu-1$ and $g_{0}^{\nu}=0$ for $v_{0}^{s}=0, s=1, \cdots, v-1$,

$$
\begin{aligned}
& v_{0}^{s}=0, s=1, \cdots, \nu-1, \\
& L_{k(d)}^{(d)} v_{0}^{\nu}=0 .
\end{aligned}
$$

Hence we can seek $v_{0}^{\nu}$ and an open set $U \subset U^{(d)}$ such that 


$$
v_{0}^{\nu} \neq 0 \text { in } U \text {. }
$$

Decompose $N\left(x, D^{\prime}\right)=\left(N^{(1)}\left(x, D^{\prime}\right), \cdots, N^{(r)}\left(x, D^{\prime}\right)\right)$ which is given by Proposition 2.2, where $N_{0}{ }^{(l)}\left(x, \xi^{\prime}\right)$ the principal part of $N^{(l)}\left(x, D^{\prime}\right)$ is generated by eigen vectors of $A\left(x, \xi^{\prime}\right)$ corresponding to $\lambda^{(l)}\left(x, \xi^{\prime}\right)$. Put

$$
u(x, \rho)=\sum_{j=0}^{M} \rho^{-j \sigma} N^{\left(l_{0}\right)}\left(x, D^{\prime}\right) Q^{\left(l_{0}\right)}(x, D)\left(\begin{array}{ccc}
\rho^{-n_{1}} & 0 \\
\ddots & \\
0 & \rho^{-n_{\nu}}
\end{array}\right) e^{i E(\rho, x)}\left(\begin{array}{c}
v_{j}^{1} \\
\vdots \\
v_{j}^{\nu}
\end{array}\right) .
$$

Then by virtue of (3.22) and (3.23) we have

$$
u(x, \rho)=e^{i E(\rho, x)}\left\{N_{0 \nu}^{\left(l_{0}\right)}\left(x, \varphi_{x^{\prime}}^{(0)}\right)\left(-\left|\varphi_{x^{\prime}}^{(0)}\right|\right)^{\nu-1} v_{0}^{\nu}(x)+0\left(\rho^{-\sigma}\right)\right\},
$$

where $N_{0 \nu}^{\left(l_{0}\right)}\left(x, \xi^{\prime}\right)$ is the $\nu$-th eigen vector of $A\left(x, \xi^{\prime}\right)$ corresponding to $\lambda^{\left(l_{0}\right)}$. Therefore it follows from (3.11) that $u(x, \rho)$ violates (3.1), if $M$ is sufficiently large and $\rho$ tends to $\infty$. Thus we have completed the proof of Theorem 3.1.

We shall here give a necessary condition in order that the Cauchy (1) for $P$, a hyperbolic system with diagonal principal part of constant multiplicity, is well posed. It seems that our condition is deeply connected with that given by Mizohata in [11].

We consider

$$
P_{t}^{s}(x, D)=\delta_{t}^{s} a(x, D)+B_{t}^{s}(x, D), \quad s, t=1, \cdots, N,
$$

where $a(x, D)=Q(x, D) q(x, D)^{\nu}, q(x, D)=D_{0}+\lambda\left(x, D^{\prime}\right)$ and $\widehat{Q}(x, \lambda$ $\left.\times\left(x, \xi^{\prime}\right), \xi^{\prime}\right) \neq 0(\widehat{Q}$ the principal part of $Q$ ) and order $a(x, D)=m$, order $B_{t}^{s} \leq m-1$.

We decompose

$$
B_{t}^{s}(x, D)=\sum_{j=0}^{m-1} B_{t j}^{s}\left(x, D^{\prime}\right) C_{t j}^{s}(x, D),
$$

where $B_{t j}^{s}$ is a pseudo-differential operator in $x^{\prime} \in R^{n}$ of order $m-1-j$ and

$$
C_{t j}^{s}(x, D)=\sum_{k=0}^{d_{t j}^{s}} C_{t j k}^{s}(x) q(x, D)^{d_{t j}^{s}-k}
$$

We note that $m-j-1+d_{t j}^{s} \leq m-1$, that is

$$
d_{t j}^{s} \leq j \text {. }
$$


We develop for a phase function $\varphi$ corresponding to $\lambda$,

$$
\begin{aligned}
e^{-i \rho \varphi} B_{t}^{s}(x, D) e^{i \rho \varphi} & =\sum \sigma_{k}\left(B_{t j}^{s}, \varphi\right) \sigma_{d_{t j}^{s}+l}\left(C_{t j}^{s}, \varphi\right) \rho^{m-j-1-k-l} \\
& =\sum \rho^{m-j-1-i} B_{t j i}^{s}(x, D)
\end{aligned}
$$

where

$$
\begin{aligned}
& B_{t j i}^{s}=\sum_{k+l=i} \sigma_{k}\left(B_{t j}^{s}, \varphi\right) \sigma_{d_{i j}^{s}+l}\left(C_{t j}^{s}, \varphi\right), \\
& \text { order } B_{t j i}^{s} \leq d_{t j}^{s}+i
\end{aligned}
$$

In particular, the principal part of $B_{t j 0}^{s}$ is given by

$$
\widehat{B}_{t j 0}^{s}(x, \xi)=\widehat{B}_{t j}^{s}\left(x, \varphi_{x}\right) C_{t j 0}^{s}(x) H(x . \xi)^{d_{t j}^{s}},
$$

where

$$
H(x, \xi)=\xi_{0}+\sum_{i=1}^{n} \lambda_{\xi_{i}}\left(x, \varphi_{x^{\prime}}\right) \xi_{i}
$$

We put

$$
E(\rho, x)=\rho \varphi(x)+\rho^{\sigma} \psi(x), \quad 0<\sigma<1 .
$$

Then we have

$$
\begin{aligned}
& e^{-i E(\rho, x)} B_{t}^{s}\left(e^{i E(\rho, x)}\right) \\
& \quad=\sum \rho^{m-j-1-i+\sigma\left(d_{t j}^{s}+i-k\right)} \sigma_{k}\left(B_{t j i}^{s}, \phi\right) \\
& =\sum \rho^{m-j-1+\sigma d_{t j}^{s}}\left(\sigma_{0}\left(B_{t j 0}^{s}, \psi\right)+0(1)\right)
\end{aligned}
$$

We set

$$
d_{j}=\max _{\pi} \sum_{s=1}^{N} d_{\pi(s) j}^{s} / N
$$

where $\pi$ stands for a permutation of $[1, \cdots, N]$. We choose the rational number $\sigma$ such that

$$
m-j-1+\sigma d_{j} \leq m-\nu+\sigma \nu
$$

for any $j$. To do so, we put

$$
\sigma=\max _{0 \leq j<\nu} \frac{\nu-j-1}{\nu-d_{j}}
$$

By (3. 24) we have $\sigma<1$. We put

$$
M_{t}^{s}=\max _{0 \leq j<\nu}\left(m-j-1+d_{t j}^{s}\right),
$$




$$
\psi_{t}^{s}=\left\{0 \leq j<\nu, m-j-1+d_{t j}^{s}=M_{t}^{s}\right\} .
$$

Then we obtain by (3.26)

$$
\max _{\pi} \sum_{s=1}^{N} M_{\pi(s)}^{s} / N=m-\nu+\sigma \nu .
$$

Volevich's lemma (c.f. [11], [12]) implies that there exist the rational numbers $m_{s}, s=1, \cdots, N$, such that

$$
M_{t}^{s} \leq m-\nu+\sigma \nu+m_{t}-m_{s}, \quad s, t=1, \cdots, N .
$$

We define $\widehat{B}_{t}^{s}(x, H)$ such that

$$
\widehat{B}_{t}^{s}(x, H)= \begin{cases}\sum_{j \in \oiint_{t}^{s}} B_{t j}^{s}\left(x, \varphi_{x}\right) C_{t j 0}^{s}(x) H^{d_{t j}^{s}} \text { if } M_{t}^{s}=m-\nu+\sigma \nu+m_{t}-m_{s}, \\ 0 \quad \text { otherwise. }\end{cases}
$$

We denote by $A(x, H)$ the matrix of which $(s, t)$-element is given by

$$
\delta_{t}^{s} Q\left(x, \varphi_{x}\right) H^{\nu}+\widehat{B}_{t}^{s}(x, H) .
$$

Then it is evident that all elements of $A(x, H)$ are polynomials in $H$ of order $\nu$. Then we have the following theorem whose proof is analogous to that of Theorem 3.1.

Theorem 3.2. Let $P$ be a hyperbolic system with diagonal principal part of constant multiplicity. If the Cauchy problem for $P$ is well posed in $G$ and $\sigma$ given by (3.27) is not zero, then all the roots with respect to $H$ of the determinant of the characteristic matrix $A(x, H)$ are zero in $G$.

Example 1. Let

$$
P=\left(\begin{array}{ll}
1 & 0 \\
0 & 1
\end{array}\right) D_{0}^{2}+\left(\begin{array}{ll}
\alpha & \beta \\
\gamma & \delta
\end{array}\right) D_{1}+\left(\begin{array}{ll}
a & b \\
c & d
\end{array}\right) D_{0}
$$

Then we have

$$
A(x, H)=\left(\begin{array}{cc}
H^{2}+\alpha & \beta \\
\gamma & H^{2}+\delta
\end{array}\right) .
$$

Hence if $P$ is well posed in $\mathbb{R}^{2}$, we obtain

$$
\alpha+\delta=0
$$




$$
\alpha \delta-\beta \gamma=0
$$

Assume $\gamma(x) \neq 0$. We put

$$
N=\left(\begin{array}{ll}
\alpha & 1 \\
\gamma & 0
\end{array}\right)
$$

Then

$$
\widetilde{P}=N^{-1} P N=D_{0}^{2}+\left(\begin{array}{ll}
0 & 1 \\
0 & 0
\end{array}\right) D_{1}+\frac{1}{\gamma}\left(\begin{array}{ll}
* & * \\
B_{1} & *
\end{array}\right) D_{0}+\frac{1}{\gamma}\left(\begin{array}{ll}
* & * \\
B_{2} & *
\end{array}\right)
$$

where

$$
\begin{aligned}
& B_{1}=\gamma D_{0} \alpha-\alpha D_{0} \gamma+\gamma(a \alpha+b \gamma)-\alpha(\alpha c+d \gamma) \\
& B_{2}=\gamma D_{0}^{2} \alpha-\alpha D_{0}^{2} \gamma+\gamma\left(a D_{0} \alpha+b D_{0} \gamma\right)-\alpha\left(c D_{0} \alpha+d D_{0} \gamma\right)
\end{aligned}
$$

Since $\widetilde{P}$ is also well posed at $\widehat{x}$, and the characteristic matrix is given by

$$
A(x, H)=\left(\begin{array}{cc}
H^{2} & 1 \\
B_{1} H & H^{2}
\end{array}\right),
$$

we have $B_{1} \equiv 0$. Moreover when $B_{1} \equiv 0$, we have

$$
A(x, H)=\left(\begin{array}{ll}
H^{2} & 1 \\
B_{2} & H^{2}
\end{array}\right) .
$$

Hence we obtain $B_{2} \equiv 0$. Thus we have (2.28) and $B_{1} \equiv B_{2} \equiv 0$ as the necessary conditions. If the rank of $\left(\begin{array}{ll}\alpha & \beta \\ \gamma & \delta\end{array}\right)$ is constant, then (2.28) and $B_{1} \equiv B_{2} \equiv 0$ is also sufficient.

Example 2. Let

$$
P=\left(\begin{array}{lll}
1 & & \\
& 1 & \\
& & 1
\end{array}\right) D_{0}+\left(\begin{array}{ccc}
0 & & \\
& 0 & \\
& & 0 \\
& & 0
\end{array}\right) D_{1}+\left(\begin{array}{lll}
b_{11} & b_{12} & b_{13} \\
b_{21} & b_{22} & b_{23} \\
b_{31} & b_{32} & b_{33}
\end{array}\right)
$$

Put

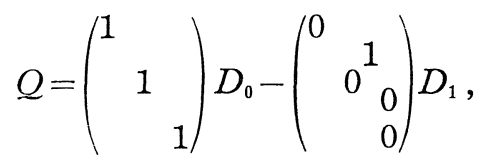

which is evidently well posed. If $P$ is well posed, 


$$
P Q=\left(\begin{array}{ccc}
1 & & \\
& 1 & \\
& & 1
\end{array}\right) D_{0}^{2}-\left(\begin{array}{lll}
0 & b_{11} & 0 \\
0 & b_{21} & 0 \\
0 & b_{31} & 0
\end{array}\right) D_{1}+B D_{0}
$$

is also well posed. Then the characteristic matrix for $P Q$

$$
A(x, H)=\left(\begin{array}{ccc}
H^{2} & b_{11} & 0 \\
0 & H^{2}+b_{21} & 0 \\
0 & b_{31} & H^{2}
\end{array}\right)
$$

Hence $b_{21}=0$ is necessary. Moreover when $b_{21}=0$, we have

$$
A(x, H)=\left(\begin{array}{ccc}
H^{2} & b_{11} & 0 \\
0 & H^{2} & b_{23} H \\
0 & b_{31} & H^{2}
\end{array}\right)
$$

Therefore $b_{31} b_{23}=0$ is necessary. Thus we obtain as the necessary condition

$$
\left\{\begin{array}{l}
b_{21}=0 \\
b_{31} b_{23}=0,
\end{array}\right.
$$

which Petkov has already derived by a different method from ours in [7]. In general, let

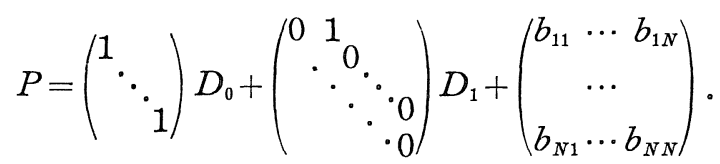

If $P$ is well posed in $\mathbb{R}^{2}$, it is necessary,

$$
\left\{\begin{array}{l}
b_{21}=0, \\
\sum_{k=3}^{N} b_{k 1} b_{2 k}=0
\end{array}\right.
$$

Example 3. Let

$$
P=\left(\begin{array}{rrrrr}
1 & & & \\
& 1 & & \\
& & 1 & \\
& & & 1
\end{array}\right) D_{0}+\left(\begin{array}{rrrr}
0 & 1 & & \\
& 0 & 0 & \\
& & 0 & 1 \\
& & & 0
\end{array}\right) D_{1}+\left(\begin{array}{cccc}
0 & 0 & 0 & 0 \\
a & b & c & d \\
0 & 0 & 0 & 0 \\
\alpha & \beta & \gamma & \delta
\end{array}\right)
$$

If $P$ is well posed, 


$$
\begin{aligned}
& a+\delta=0, \\
& a \delta-\alpha c=0, \\
& \alpha(a b+\alpha d)-a(a \beta+\alpha \delta)+2\left(\alpha D_{0} a-a D_{0} \alpha\right)=0, \\
& \alpha\left(b D_{0} a+d D_{0} \alpha\right)-a\left(\beta D_{0} a+\delta D_{0} \alpha\right)+\alpha D_{0}^{2} a-a D_{0}^{2} \alpha=0,
\end{aligned}
$$

are necessary conditions. If the rank of $\left(\begin{array}{ll}a & c \\ \alpha & \delta\end{array}\right)$ is constant, these conditions are sufficients.

\section{References}

[1] Chazarain, J., Opérateurs hyperboliques a caractéristiques de multiplicité constante, Anal. de l'Institut Fourier, 24 (1974), 173-202.

[2] Flaschka, H. and Strang, G., The correctness of the Cauchy problem, Adv. in Math., 6 (1971), 347-379.

[3] Gourdin, D., Systèmes faiblement hyperboliques, Seminaire Goulaouic-Schroartz, 1975-1976.

[4] Kajitani, K., Strongly hyperbolic systems with variable coefficients, Publ. RIMS Kyoto Univ., 9 (1974), 597-612.

[5] Leray, J. and Ohya, Y., Systèmes lineair hyperboliques non-stricts, Colloque de Liege, (1964), C. N. R. B.

[6] Mizohata, S. and Ohya, Y., Sur la condition d'hyperbolicité pour les équations a caractéristiques multiples I, II, Publ. RIMS Kyoto Univ., 4 (1968), 511-526, Japanese J. Math., 40 (1971), 63-104.

[7] Petkov, V. M., Equations et systèmes hyperboliques a caractéristiques multiples, Laboratoire d'Analyse Numerique, (1975).

[8] Petkov, V. M., Une parametrix du problème de Cauchy pour des systèms hyperboliques a caractéristiques de multiplicité constante. C. R. Acad. Sc. Burgare, 29 (1976), 1095-1097.

[9] Vaillant, J. et Bersin, Paramétrix du problèmes de Cauchy pour un système faiblement hyperbolique à caractéristiques multiples, R., C. R. Acad. Sc. Paris, (1976), 485-487.

[10] Yamahara, H., On the Cauchy problem for weakly hyperbolic systems, Proc. Japan Acad., 52 (1976), 167-170.

[11] Mizohata, S., On Kowalewskian systems, Russian Math. Surveys, 29 (1975), 223235.

[12] Volevich, L. R., On general systems of differential equations, Soviet Math. Dokl., 1 (1960), 458-461. 